\title{
Accounting for vibronic features through a mixed quantum-classical scheme: structure, dynamics and absorption spectrum of a perylene diimide dye in solution
}

\author{
Alekos Segalina $^{a}$, Javier Cerezo $^{b}$, Giacomo Prampolini ${ }^{c, *}$ \\ Fabrizio Santoro ${ }^{c, *}$ and Mariachiara Pastore ${ }^{a, *}$ \\ ${ }^{a}$ Université de Lorraine \& CNRS, \\ LPCT, UMR 7019, F-54000 Nancy, France \\ ${ }^{b}$ Departamento de Química and Institute for Advanced Research in Chemical Sciences (IAdChem), \\ Universidad Autónoma de Madrid, 28049 Madrid, Spain \\ ${ }^{c}$ Istituto di Chimica dei Composti Organo Metallici, Consiglio Nazionale delle Ricerche, (ICCOM-CNR) \\ SS di Pisa, Area della Ricerca, via G. Moruzzi 1, I-56124 Pisa, Italy
}

October 9, 2020

*corresponding authors: giacomo.prampolini@pi.iccom.cnr.it ; fabrizio.santoro@pi.iccom.cnr.it and mariachiara.pastore@univlorraine.fr 


\begin{abstract}
The optical absorption spectrum of a perylene diimide (PDI) dye in acetonitrile solution is simulated by using the recently developed (J. Chem. Theory Comput. 2020, 16, 1215 - 1231) $A d-M D \mid g V H$ method. This mixed quantum-classical (MQC) approach is based on an adiabatic (Ad) separation of soft(classical)/stiff(quantum) nuclear degrees of freedom, and expresses the spectrum as a conformational average (over the soft coordinates) of vibronic spectra (for the stiff coordinates) obtained through the Generalized Vertical Hessian $(g \mathrm{VH})$ vibronic approach. The average is performed over snapshots extracted from classical Molecular Dynamics (MD) runs, performed with a specifically parameterized Quantum-Mechanically Derived Force Field (QMD-FF). A comprehensive assessment of the reliability of different approaches, designed to reproduce spectral shapes of flexible molecules, is here presented. First, the differences in the sampled configurational space and their consequences on the prediction of the absorption spectra are evaluated by comparing the results obtained by means of the specifc QMD-FF and of a general-purpose transferable FF with those of a reference ab initio MD (AIMD) in gas phase, in both a purely-classical scheme (ensemble average) and in the $A d-M D \mid g V H$ framework. Next, classical ensemble average and MQC predictions are also obtained for the PDI dynamics in solution, and compared with the results of a "static" approach, based on vibronic calculations carried out on a single optimized perylene diimide structure. In the classical ensemble average approach, the remarkably different samplings obtained with the two FFs lead to sizeable changes in both position and intensity of the predicted spectra, with the one computed along the QMD-FF trajectory closely matching its AIMD counterpart. Conversely, at $A d-M D \mid g V H$ level of theory, the different samplings deliver very similar vibronic spectra, indicating that the error found in the absorption spectra obtained with the general-purpose FF mainly concerns the stiff modes, since it can be effectively corrected by the quadratic extrapolation performed by $g \mathrm{VH}$ to locate the minima of the ground and excited state potential energy surfaces along such coordinates. Furthermore, in the perspective of studying the self-assembling process of PDI dyes and the vibronic spectra of large-sized aggregates, the use of a molecule-specific QMD-FF also appears mandatory, considering the significant errors found in the GAFF trajectory in the flexible lateral chain populations, which dictate the supramolecular aggregation properties.
\end{abstract}

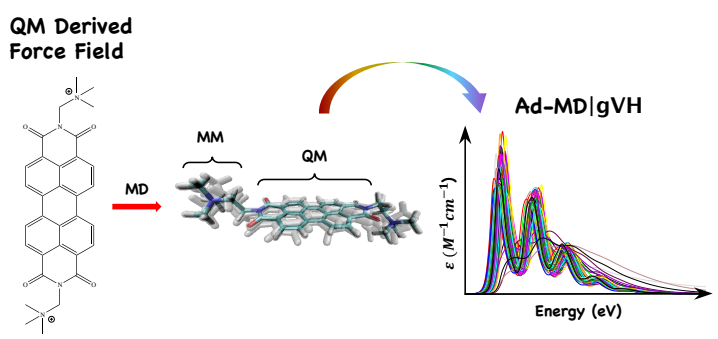

TOC 


\section{Introduction}

Perylene diimides (PDIs) dyes, Figure 1, panel a), have been widely employed as photoactive materials, thanks to their notable chemical, thermal and photochemical stability, wide and in-

a)

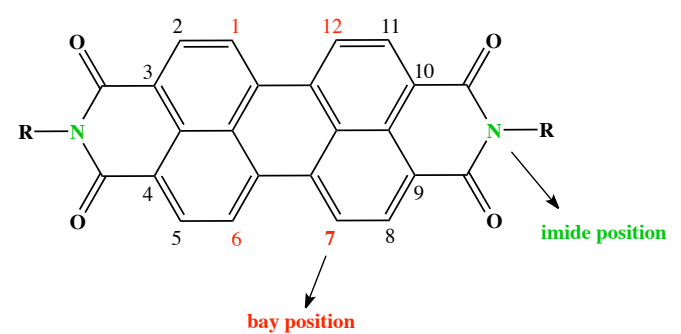

b)

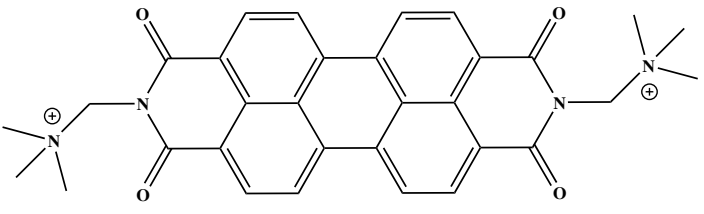

Figure 1: a) Chemical structure of a generic PDI sensitizer: the bay and imide positions are evidenced in red and green, respectively. b) N,N $\mathrm{N}^{\prime}$-bis(2-(trimethylammonium)-ethylene)-perylene-3,4,9,10-tetracarboxylic acid bis-imide PDI molecule investigated in this work.

tense optical absorption in the visible to near-infrared spectral window and excellent charge transport properties. ${ }^{1-10}$ Moreover, PDIs derivatives, in virtue of their tendency to form cofacial H-aggregates, ${ }^{11}$ easily undergo self-assembling in ordered 1D nanostructures through hydrophobic/hydrophilic and $\pi$ - $\pi$ stacking interactions. ${ }^{12-14}$ On the one hand, side chains (R substituents at the imide position, see Figure 1) control the solubility of PDIs derivatives, and can be used to optimize the molecular packing conformation, hence modulating the color and other physico-chemical properties of the PDI aggregates. ${ }^{15}$ On the other hand, these side chains are not expected to largely affect the absorption and emission properties, because the HOMO and LUMO orbitals, which describe the $S_{0} \rightarrow S_{1}$ transition in a single particle picture, present nodal planes at the imide nitrogens. ${ }^{14,16}$ Nonetheless, modulation of the optical properties of monomers can be efficiently obtained, when PDIs are substituted at the aromatic core, in the bay positions (1, 6, 7 and 12 evidenced in Figure 1). ${ }^{15,17}$

The lowest-energy excited state of a PDI monomer is usually energetically well separated from the other bright transitions and presents an absorption maximum around $530 \mathrm{~nm}$ with a strong vibronic progression. The intensity reversal between the $0 \rightarrow 0$ and $0 \rightarrow 1$ vibronic bands is generally used as spectral signature of the formation of PDI-aggregates in solution or on semiconductor surfaces. ${ }^{10,18,19}$ Even though these aggregates are characterized by a complex pho- 
tophysics, some authors have suggested the idea that both localized excimers and low-energy charge transfer (CT) states take part to the excited state relaxation dynamics, with a decisive role of the packing arrangement in driving the process. ${ }^{20-25}$ After the first example by Fulton and Gouterman ${ }^{26,27}$ of numerically introducing vibronic coupling in aggregates models, a large number of studies has investigated the impact of vibronic coupling on molecular assemblies photophysics. $^{22,28-43}$

From a theoretical and computational point of view, the route to an accurate description of the spectral vibronic features in large-sized molecular aggregates presents a number of challenging tasks: i) the configurational space sampling of the multi-chromophoric assembly ground state; ii) the treatment of both solute-solute and solute-solvent interactions, which might require to be included explicitly, to account for strong and local phenomena as for instance Hydrogenbonds; iii) the inclusion of inter- and intra-molecular quantum vibronic effects in the calculation of the absorption spectra; iv) the necessity of considering the coupling between multiple exciton and/or CT quasi-degenerate excited states, participating in the aggregate's spectrum. ${ }^{16}$

Tasks i) and ii) can be tackled resorting to a classical (CL) description of the nuclear dynamics, which allows for the simulation of the wide time and length scale required to observe self-assembly phenomena. Yet, as far as $\pi$-conjugated molecules are concerned, care should be taken in selecting a proper force-field (FF) parameter set, capable to accurately describe both monomer flexibility and intermolecular interactions, eventually leading to a reliable representation of the $\pi-\pi$ driven aggregation. This might introduce a further obstacle, which can be overcome by refining or fully re-parameterizing the FF, based on accurate Quantum Mechanical (QM) data, as recently reported by several groups. ${ }^{44-49}$ Once the reliability of the classical MD trajectories has been validated, a very convenient method to compute spectra of complex and flexible systems in explicit environments is performing a Classical Ensemble Average of Vertical Excitations (CEA-VE). It essentially consists in performing a sufficiently long and accurate MD trajectory, from which a number of uncorrelated snapshots, large enough to ensure convergence, is extracted, and successively computing vertical transition energies and intensities for each frame. In this framework, more sophisticated samplings and combination with clustering techniques have been proposed. ${ }^{50-52}$ Within the CEA-VE framework, the explicit 
solute-solvent and solute-solute interactions mentioned in ii) can be accounted for straightforwardly, for example with a quantum mechanic:molecular mechanic (QM:MM) scheme. ${ }^{53}$

Quantum nuclear effects are responsible for vibronic peaks described above but, they also impact remarkably structureless bands, modifying the spectral maximum and increasing its width. ${ }^{54-56}$ The most popular implementations of CEA-VE approach neglect all these effects, although some of them can be introduced, at the cost to assume harmonic approximation, sampling the Wigner distribution within the so called nuclear ensemble approach. ${ }^{57,58}$ However, the vibronic structures described above for PDI and its aggregates can only be simulated with a fully quantum description of nuclear motion. Finally, tackling task iv) to achieve a proper description of the effect of inter-state couplings among quasi-degenerate states requires the adoption of quantum dynamical methods based on wavepacket propagations on the coupled potential surfaces. To the best of our knowledge, however, there is no well established methodology merging all these capabilities in a unique computational protocol.

In the last two decades several time-independent ${ }^{59-63}$ and time-dependent (TD ${ }^{64-69}$ methods have been proposed to compute fully quantum spectra, but for large systems they basically rely on harmonic approximation for the potential energy surfaces and therefore they are suited for rigid systems rather than for flexible ones. ${ }^{59-64,67,68}$ On these grounds, a number of mixed quantum classical (MQC) approaches -where quantum and classical refers to the way the nuclear motion is treated- have been proposed. ${ }^{56,70-74}$ We recently introduced the "Adiabatic Molecular Dynamics generalized Vertical Hessian", $A d-M D \mid g V H,{ }^{56}$ and is aimed to simulate the electronic spectra of flexible dyes in condensed phase, yet preserving their vibronic features. At variance with most of the other approaches, ${ }^{71-73,75}$ rather than adopting the standard solute-solvent partition scheme, the $A d-M D \mid g V H$ method is based on differentiating the nuclear degrees of freedom in stiff and soft modes, and assuming that the former modes are much faster than the latter (Adiabatic hypothesis). In this framework, the spectrum can be rigorously obtained as an average of configuration-specific vibronic spectra, computed along the $M D$ trajectories sampling a selected set of large amplitude soft modes. For dyes with negligible inter-state couplings, each vibronic spectrum might be retrieved by means of the $\mathrm{gVH}$ approach, ${ }^{56}$ a generalization of the Vertical Hessian model. ${ }^{76,77}$ Conversely, in order to be able to 
deal with spectra of molecular aggregates properly accounting for task iv), $A d-M D \mid g V H$ needs to be generalized substituting the engine that computes the spectra, i.e. the $g V H$ approach, with a methodology able to account for strong inter-state couplings.

Before any attempt to generalize the theoretical framework to obtain an accurate description of the vibronic spectra of large-sized self-assembled PDIs, it is necessary to extensively investigate the protocol performances on monomers in solution. To this end, two points require, in our opinion, particular attention: (a) the capability of the adopted FF to properly describe both the rigidity of PDI core and the flexibility of its lateral chains (which are expected to play an important role in the aggregation mechanism), and, (b) the accuracy of the $A d-M D \mid g V H$ method in combination with the selected FF in properly reproducing the well-resolved vibronic structure of the spectrum of a solvated PDI monomer. In this work, the $A d-M D \mid g V H$ method will be therefore applied in combination with a molecule-specific QM derived FF (QMD-FF), to obtain the absorption spectrum of the $\mathrm{N}, \mathrm{N}^{\prime}$-bis (2-(trimethylammonium)-ethylene)-perylene3,4,9,10-tetracarboxylic acid bis-imide monomer (displayed in the (b) panel of Figure 1) in acetonitrile $(\mathrm{ACN})$, eventually validating the results through the comparison of the computed spectra with their experimental counterparts. ${ }^{10}$ Besides setting up a sound MQC protocol to successively tackle vibronic spectra of large $\pi$-aggregates in solution, this work is also the occasion to present an extensive evaluation of the performance of a number of different techniques, designed to reproduce spectral shapes of flexible molecules, whose interest goes beyond the specific PDI system. In order to perform such an extended analysis, we designed a computational protocol that is schematically displayed in Figure 2. More in detail, we will first compare the predictions of our QMD-FF, parameterized on the basis of Density Functional Theory (DFT) data specifically computed for the target PDI, to the results of a general-purpose, transferable FF, investigating the differences in terms of the sampled configurational space and its consequences on the prediction of purely-classical CEA-VE spectra. To have a reference to compare with and assess the FFs capability in delivering a reliable description of the monomer structure and dynamics, we also performed calculations in gas phase, benchmarking the results with those obtained by an ab initio MD (AIMD) simulation. Next, we investigate the difference among the two MD and the AIMD samplings, when combined with the $A d-M D \mid g V H$ method 


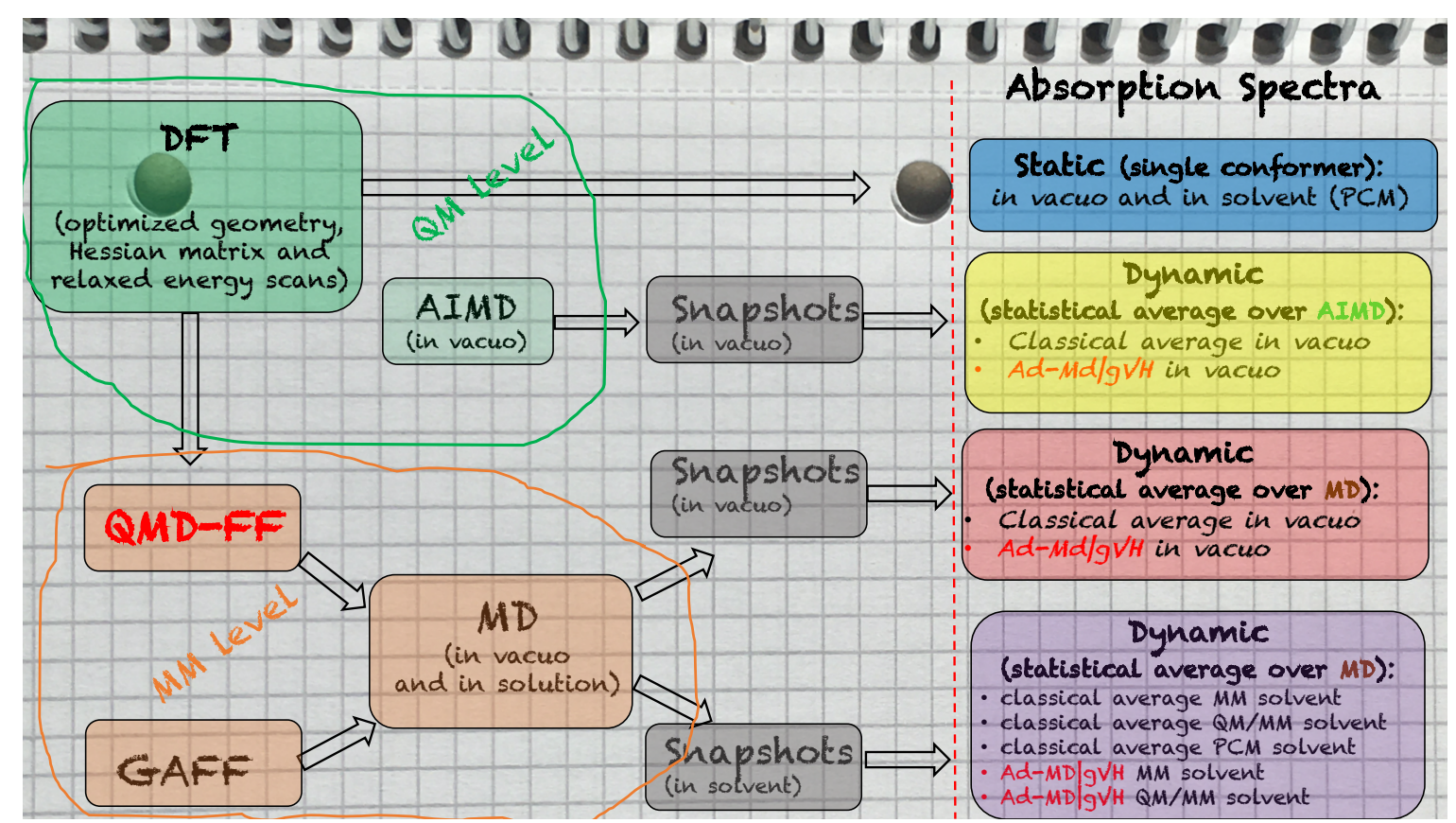

Figure 2: Flow chart of the MQC approach presented in this work: the integration of QM (green) and CL (MM, orange) techniques leads to the simulation of absorption spectra (right panels) at different level of complexity, thus allowing to unravel selected effects acting on spectral shape, as the system dynamics, the presence of the solvent or the vibronic features. The key ingredients for the most complete description, i.e. the accurate and specific QMD-FF and the recently proposed $A d-M D \mid g V H$ method, are highlighted in red.

to account for vibronic progressions. Finally, we compare the MQC predictions, which account for the solvated PDI dynamics, with the results of a standard "static" approach, based on vibronic calculations carried out upon harmonic approximation on a single optimized PDI conformer, accounting for the solvent with a polarizable continuum model (C-PCM). ${ }^{78}$ The paper is organized as follows: after a concise description of the MQC protocol in Section 2, we give the computational details in Section 3 and discuss all results in Section 4; our main conclusions are drawn in Section 5. 


\section{The MQC protocol}

The MQC protocol presented in this work consists in the hierarchical integration of different QM and MM techniques: a QMD-FF parameterization of the target solute based on QM data, MD simulations carried out with the resulting FF and, eventually, the calculation of the absorption spectra along the MD sampled trajectories. As far as the FF ruling PDI's flexibility $\left(E_{P D I}^{\text {intra }}\right)$ is concerned, a specific QMD-FF intra-molecular FF is here parameterized according to the JOYCE protocol, ${ }^{79-81}$ using the DFT database purposely computed for the target molecule, which contains the target PDI's optimized geometry, the equilibrium Hessian matrix and the most relevant relaxed energy scans. A detailed description of the JOYCE parameterization procedure can be found in Section 1 of the Supporting Information or in the original papers. ${ }^{79,80}$ Extensive MD runs, either in gas phase or solution, are then carried out exploiting the QMD-FF, to accurately sample PDI's conformational space through a reliable collection of system snapshots. Finally, the absorption spectra is obtained by applying the $A d-M D \mid g V H$ method over the aforementioned frame collection as described in the following.

In a TD formalism, the general QM expression of the absorption lineshape $L^{Q M}(\omega)$ from an initial electronic state $i$ to a final one, $f$, is

$$
L^{Q M}(\omega)=\frac{1}{2 \pi Z_{v_{i}}} \int \operatorname{Tr}\left[\boldsymbol{\mu}_{i f} e^{-i t H_{f} / \hbar} \boldsymbol{\mu}_{f i} e^{-(\beta-i t / \hbar) H_{i}}\right] e^{i \omega t} d t
$$

$H_{i}$ and $H_{f}$ are the Hamiltonians for the $i$ and $f$ states, $\boldsymbol{\mu}_{i f}$ their transition electric dipole moment (with $\boldsymbol{\mu}_{i f}=\boldsymbol{\mu}_{f i}$ ) and $\operatorname{Tr}$ denotes the trace operation. $\beta=\left(K_{B} T\right)^{-1}$, where $K_{B}$ is the Boltzmann constant, $\mathrm{T}$ the absolute temperature, and $Z_{v_{i}}$ is the partition function of the initial vibrational states. From equation (1), it is possible to obtain a CL approximation to the spectrum, $L^{C L}(\omega)$, in two steps. First, following Lax, ${ }^{82}$ we neglect the commutators between the Hamiltonians $H_{i}$ and $H_{f}$ and with the operator $\boldsymbol{\mu}_{i f},{ }^{56}$ and, second, the quantum distribution of the system in the initial electronic state, $\rho_{i}^{Q M}(\mathbf{Q}, T)$, is approximated with its CL analogue, $\rho_{i}^{C L}(\mathbf{Q}, T)$, which can be sampled along the MD trajectory. ${ }^{56}$ In this way we obtain the CEA-VE spectral lineshape

$$
L^{C L}(\omega)=\frac{1}{N_{\text {con }}} \sum_{\alpha}\left|\boldsymbol{\mu}_{i f}\left(\mathbf{Q}^{\alpha, C L}\right)\right|^{2} g\left(\omega-\Delta \Omega\left(\mathbf{Q}^{\alpha, C L}\right)\right)
$$

where $g$ is a linewidth function, in condensed phase usually a Gaussian, whose width can be set 
to account phenomenologically for a number of effects missing in the model (like the quantum nature of the nuclear motion).

To obtain the MQC spectral shape, $L^{M Q C}(\omega)$, we here resort to our recently proposed $A d-M D \mid g V H$ method. ${ }^{56}$ According to the $A d-M D \mid g V H$ model, all nuclear degrees of freedom (DoFs) of the solute+solvent system can be separated in two categories: stiff modes, $\mathbf{r}$, pertaining to the dye (and to a number of selected solvent molecules, if required), and the soft modes, $\mathbf{R}$, represented by the flexible DoFs of the dye together with all the remaining environmental modes. The former $\mathbf{r}$ set is handled at QM level, while the $\mathbf{R}$ collection is treated at CL level. Concretely, as for the CEA-VE spectra, the CL configurational space $\rho_{i}^{C L}([\mathbf{R}, \mathbf{r}], T)$ is first sampled through a representative number $N_{\text {con }}$ of MD snapshots $\left(\mathbf{R}^{\alpha}, \mathbf{r}^{\alpha}\right)$. Next, in order to (re-)introduce a QM vibronic treatment of the stiff-coordinates $\mathbf{r}^{\alpha}$, we invoke an adiabatic approximation, thus assuming that $\mathbf{R}$ coordinates are much slower than stiff ones, which can hence rearrange very quickly to any $\Delta \mathbf{R}$ displacement, so that the soft $\mathbf{R}$ coordinates can be considered frozen at each sampled frame $\alpha$. This allows us to build, at each $\alpha$, reduced-dimensionality harmonic potential energy surfaces (PES) along the stiff-coordinates, for the initial $\left(V_{i}\left(\mathbf{r} ; \mathbf{R}^{\alpha}\right)\right)$ and final-state $\left(V_{f}\left(\mathbf{r} ; \mathbf{R}^{\alpha}\right)\right)$. In order to define $V_{k}\left(\mathbf{r} ; \mathbf{R}^{\alpha}\right)(k=i, f)$, at each $\alpha$ snapshot we compute with a QM:MM scheme the energy gradient and Hessian of the initial and final state for a subset of the system coordinates, comprising all dyes atoms and a first layer of environment molecules. Soft modes are projected out moving to a set of redundant curvilinear internal coordinates ${ }^{77,83}$ and the applying iteratively proper projectors. Further details can be found in the original paper. ${ }^{56} V_{i}\left(\mathbf{r} ; \mathbf{R}^{\alpha}\right)$, and $V_{f}\left(\mathbf{r} ; \mathbf{R}^{\alpha}\right)$ PES can be eventually exploited to compute, through equation (1), a vibronic quantum spectrum $L_{\alpha}^{Q M, \mathbf{r}}(\omega)$, which involves the fast coordinates $\mathbf{r}$ only, and is specific for each sampled CL configuration $\mathbf{R}^{\alpha}$. Then, $L^{M Q C}(\omega)$ then is simply the average of all the reduced-dimensionality vibronic spectra, $L_{\alpha}^{Q M, \mathbf{r}}(\omega) .{ }^{56}$

$$
L^{M Q C}(\omega)=\frac{1}{N_{\text {con }}} \sum_{\alpha} L_{\alpha}^{Q M, \mathbf{r}}(\omega)
$$

Finally, it might be worth mentioning that the MQC equation (3) and the CL one (2) are formally very similar. The fundamental difference is that the phenomenological lineshape $g$, required by the CL lineshape $L^{C L}(\omega)$ and with the same Gaussian shape for each sampled configuration $\alpha$, 
is substituted in $L^{C L}(\omega)$ by the $\alpha$-specific $L_{\alpha}^{Q M, r}(\omega)$, i.e. without introducing any phenomenological factor. In summary $A d-M D \mid g V H$ treats at quantum harmonic level the stiff DoFs and at anharmonic but classical level the soft DoFs (and, although in an approximate way, also the coupling between soft and stiff DoFs, since $L_{\alpha}^{Q M, \mathbf{r}}(\omega)$ depend on $\alpha$ ). A full quantum anharmonic treatment for large molecules is still out of the reach, although promising approaches have been proposed when anharmonicty is confined to few modes. ${ }^{84}$ Anharmonic effects along stiff DoFs could be included within $A d-M D \mid g V H$ by moving them to the "classical" set. Furthermore, this approach could be combined with a path-integral dynamic (instead of the classical MD), as recently proposed in order to account for some nuclear quantum effects (but not vibronic resolution). ${ }^{72,85}$

\section{Computational Details}

\subsection{MM calculations}

Two different FFs have been tested in this work. First, the very popular, general-purpose, generalized AMBER force field (GAFF) ${ }^{86}$ was built by using the antechamber program available in the AMBER16 package ${ }^{87}$ and adopting the RESP protocol for the point-charges calculated using the HF/6-31G* level of theory. Then, all the GAFF topology files were transformed into GROMACS-type files by exploiting the ACPYPE - AnteChamber PYthon Parser interface program. ${ }^{88}$ All PDI's intermolecular FF parameters, and those concerning the solvent (ACN) and the counter ion $\left(\mathrm{Cl}^{-}\right)$where also transferred from GAFF. ${ }^{89}$ All GAFF parameters are reported

in the Supporting Information (see Tables A to E) or can be found in the original papers. ${ }^{86,89}$

The second FF is conversely a QMD-FF, parametrized with respect to the DFT data for the isolated PDI molecule. All intra-molecular PDI's parameters were obtained with the JOYCE code, ${ }^{90}$ carried out by minimizing the JOYCE objective function I:

$$
I^{\text {intra }}=\sum_{g}^{N_{\text {geom }}} W_{g}\left[\left[\Delta U-E_{P D I}^{\text {intra }}\right]_{g}\right]^{2}+\sum_{K \leq L}^{3 N-6} W_{K L}^{\prime}\left[H_{K L}-\left(\frac{\partial^{2} E_{P D I}^{\text {intra }}}{\partial Q_{K} \partial Q_{L}}\right)\right]_{g=0}^{2}
$$

where $W_{g}$ and $W_{K L}^{\prime}$ terms are the user defined weights, the first sum runs over the PDI $g$ conformations considered in the QM relaxed scans, and $\Delta U$ is the QM internal energy. The second 
sum runs over the QM normal modes, where $H_{K L}$ is the QM Hessian matrix evaluated at the equilibrium geometry ( $g=0$ ), while $Q_{K}$ is the $K^{\text {th }}$ normal coordinate. Further details about the parameterization are included in Section 1.2 of the Supporting Information, where the parameters are collected in Tables A to E, or can be found in the original papers. ${ }^{79-81}$ The LJ parameters entering the PDI's intermolecular term (S4) were instead transferred from the OPLS libraries as implemented in the GROMACS5.1 package, ${ }^{87}$ whereas the point-charges were obtained again through the RESP protocol by using the Antechamber suite. Finally, for consistency with the PDI intermolecular parameter set, all $\mathrm{ACN}$ and $\mathrm{Cl}^{-}$parameters were also taken from the OPLS FF.

Classical MD simulations for both the isolated PDI molecule in gas phase (GP) and the solvated system in ACN solution (PDI@ACN) were performed by exploiting the GROMACS5.1 Engine. ${ }^{87}$ In GP, both FFs have been employed in the NVT ensemble for $10 \mathrm{~ns}$, by imposing a timestep (ts) of $0.1 \mathrm{fs}$ and a temperature of $300 \mathrm{~K}$, through the use of the Berendsen thermostat. ${ }^{91}$ Turning to condensed phase, a PDI@ACN system, composed of the PDI solute, two $\mathrm{Cl}^{-}$counterions, and $\sim 1000 \mathrm{ACN}$ molecules, was initially minimized to remove bad contacts and thereafter thermally equilibrated for $\sim 2 \mathrm{~ns}$ at $300 \mathrm{~K}$ in the NVT ensemble. The final NPT production runs were carried out at 1 atm and $300 \mathrm{~K}$ through the Parrinello-Raman ${ }^{92}$ and the v-rescale ${ }^{93}$ schemes by using coupling constants of 0.1 ps and 1 ps respectively and applying periodic boundary conditions. Also for the simulations of the PDI@ACN we set the timestep to $0.1 \mathrm{fs}$ and the cutoff radius for the Coulombic charge-charge and LJ terms to $11 \AA$ whereas we accounted for the long-range electrostatics interactions by means of the particle mesh Ewald (PME) procedure.

All the MD trajectories were stored in sets of $100 \alpha$-conformations, equally spaced in terms of time, and used first to investigate the statistical behaviour of key structural parameters, and, next, to compute the final spectra. Furthermore, in order to evaluate the extent of the first solvation layer, we analyzed the MD runs to compute the atomic pair correlation functions, $g_{\alpha \beta}(r)$, between selected $\alpha$ and $\beta$ atoms, of the PDI solute and the solvent molecules, respectively. 


\subsection{QM calculations}

QM static calculations were carried out with GAUSSIAN $16^{94}$ package, whereas the Terachem software $^{95-97}$ was employed for the GP AIMD run. For all the DFT/TD-DFT calculations discussed here the CAM-B3LYP functional ${ }^{98}$ in combination with the $6-31 \mathrm{G}^{*}$ basis set was adopted.

We note that, despite slight differences regarding implementation details and default computational settings adopted are expected between Gaussian and Terachem calculations, we have checked that both codes provide negligible variations in both the computed energy (about 0.6 $\mathrm{kJ} / \mathrm{mol}$ ) and optimized geometries $(\mathrm{RMSD} \simeq 0.05 \AA$ ), thus ensuring a trustful comparison. To obtain the data for the QMD-FF parameterization, the PDI molecule ground state was fully optimized and the resulting geometry stored together with its Hessian matrix. The Grimme's Dispersion DFT-D3 correction ${ }^{99}$ was also adopted: notwithstanding its effect is expected to be negligible on the isolated PDI monomer, reliably accounting for dispersion interactions may become decisive when investigating $\pi$-aggregates, which is the future goal of our work. Additionally, a number of relaxed torsional energy scans were also carried out at the same level of theory, optimizing all coordinates except the scanned flexible chain dihedrals, $\delta_{\mu}$, entering in equation (S7). To partially screen the electrostatic repulsion of the lateral chains due to the +2 charge of the PDI molecule, both geometry optimization and relaxed scans were carried out in an implicit solvent adopting the conductor-like polarizable continuum model (C-PCM). ${ }^{78}$

To retrieve the energy, gradients and Hessian matrix in initial (ground) and final (excited) PDI's electronic states, needed for the simulation of the vibronic absorption spectra within the $A d-M D \mid g V H$ formalism, DFT and TD-DFT calculations were, respectively, carried out, either for the GP or PDI@ACN system, at each configuration extracted from the MD trajectories. CAM-B3LYP/6-31G* AIMD simulations have been performed on the isolated PDI in GP starting from the optimized structure of PDI, which has been obtained at the same level of theory. The AIMD simulations have been conducted for 103 ps in the NVT ensemble, where the first 3 ps have been used for equilibration and hence discarded, using a timestep of $0.1 \mathrm{fs}$ and setting the temperature to $300 \mathrm{~K}$, by using the Bussi-Parrinello Langevin scheme and imposing a damping time of $200 \mathrm{fs}$. In the following, the AIMD trajectory will be labeled as "DFT". 


\subsection{Absorption Spectra}

\section{Embedding schemes}

Absorption spectra were computed for both the isolated monomer and the solvated PDI@ACN system. For the former system, GP MD (GAFF and JOYCE) and AIMD (DFT) trajectories were employed. Conversely, to account for the embedding effects, several protocols of increasing complexity were applied to each MD snapshot extracted from the PDI@ACN runs:

i) C-PCM: All the ACN molecules and $\mathrm{Cl}^{-}$counterions are removed from each frame, and the solvent effects accounted for at C-PCM level, considering ACN and the less polar diethyl ether (see Supporting Information).

ii) EEpc: Following an Electronic Embedding (EE) scheme, all the solvent molecules and counterions within a large cut-off radius of $20 \AA$ from the PDI center of mass are included in the calculation as point-charges (pc).

iii) QM:MM/EE: The embedding environment is included according to a mixed QM:MM/EE scheme, where the QM:MM model was built according to the ONIOM approach, considering the solute in the model system (high layer) and by including the first solvation shell, determined by the $g_{\alpha \beta}(r)$ correlation functions, into the real system. The latter is described at MM level making use of the same parameters obtained for the GAFF or JOYCE FFs. Finally, the EE scheme consisted as usual in including all ACN and $\mathrm{Cl}^{-}$ atoms within the large $20 \AA$ radius as point charges.

iv) QM/EE:The solvent molecules and the counterions are treated using a mixed QM/EE approach, for which the solvent molecules of the first solvation layer are explicitly included in the KS Hamiltonian, while the remaining solvent molecules, within a $20 \AA$ radius, are included as point charges.

\section{CEA-VE spectra}

CEA-VE spectra are obtained, for both GP and PDI@ACN systems, by computing the vertical 
transition energies and intensities over the 100 snapshots sets extracted either from the GAFF, JOYCE or DFT trajectories. The final spectral shape is retrieved according to equation (2), using a phenomenological convolution with a Gaussian functions having with HWHM=0.05 eV. For the solvated system, C-PCM, EEpc and QM/EE schemes were separately employed for both FFs.

\section{Ad - MD $\mid$ gVH spectra}

All the configuration-specific vibronic spectra, necessary for the $A d-M D \mid g V H$ calculations, have been obtained for both GP and PDI@ACN systems in Franck-Condon approximation, with $\mathscr{F} \mathscr{C}$ classes 3.0, ${ }^{100,101}$ available upon request as $\beta$ release. For each snapshot, the code computes the vibronic spectrum at $300 \mathrm{~K}$ with the TD formulation. ${ }^{64-69,102}$ To this end it reads energies, gradients and Hessian of the initial and final electronic states computed over the snapshot sets, and builds up the reduced dimensionality $g V H$ model by projecting out the soft modes. The spectra for the different snapshots are then averaged to obtain the final $A d-M D \mid g V H$ spectrum according to equation (3). It accounts explicitly for possible broadening mechanisms, like the inhomogeneous one, hence, in principle, it does not require the adoption of any phenomenological Gaussian. In practice, since the sum over conformations, $\alpha$, is necessarily finite, the average arising from Eq. 3 may display some noise. Therefore, in order to compensate the limited conformational sampling and obtains smooth profiles, $L_{r}^{\alpha, q}(\omega)$ spectra are convoluted

with a narrow a Gaussian with $\mathrm{HWHM}=0.01 \mathrm{eV}$. We showed in ref. ${ }^{56}$ that this procedure only removes the noise but does not alter the overall shape and width and of the spectrum. As far as the PDI@ACN system is concerned the solvation scheme QM:MM/EE was adopted since we recently demonstrate the necessity to use a three-layers set up in order to minimize the spurious imaginary frequencies. In particular, the LJ interactions, that are included in the treatment of the first solvation layer, were found fundamental to properly polarize the QM wave-function. The effect of the mutual solute/solvent polarization on the transition energies was accounted with a mixed approach in which vertical excitations are computed with the QM/EE scheme, including the first solvation shell in the QM region, while the first and second energy derivatives are obtained from the QM:MM/EE scheme. For sake of simplicity, in the context of vibronic spectra, in the following, we will refer to this mixed approach as QM/EE. 


\section{Results}

\subsection{PDI structure and dynamics}

\subsubsection{Gas phase}

Depending on the relative orientation of the aliphatic side chains with respect to the perylene's plane, DFT optimizations indicate the presence of two possible minima, corresponding to the syn or to the anti conformer (left a) panel in Figure 3). These isomers are almost degenerate,

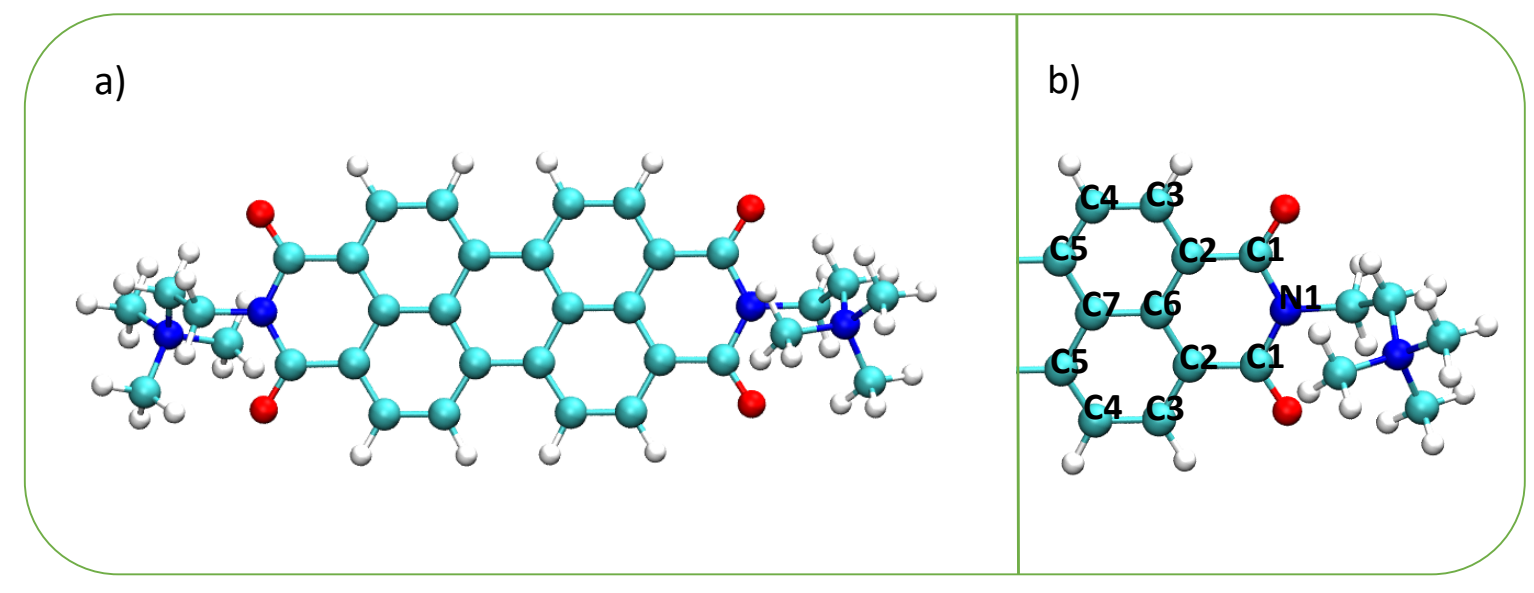

Figure 3: a) DFT optimized structure of the PDI anti-conformer; b) Atom labels used in QMD-FF parameterization for the PDI aromatic core.

with an energy difference of $\sim 0.1 \mathrm{~kJ} / \mathrm{mol}$, and, besides the relaxed scans, only the Hessian matrix of the anti conformer was considered to build the QMD-FF.

Figure 4 compares the torsional relaxed energy scans computed at DFT level with the corresponding MM scans, obtained by using either the GAFF of the JOYCE FF. As expected, the torsional profiles obtained by the QMD-FF better compare with DFT with respect to the ones calculated using GAFF. In particular, for $\delta_{1}$ and $\delta_{2}$, the most stable minima predicted by GAFF $\left(90^{\circ}\right.$ and $180^{\circ}$, respectively) correspond to maxima $\left.\left(\delta_{1}\right)\right)$ or higher energy local minima $\left.\left(\delta_{2}\right)\right)$ of the QM torsional profile and, in general, the energy barriers between the different minima are underestimated, thus favoring the inter-conversion between the syn and ant $i$ conformers. 

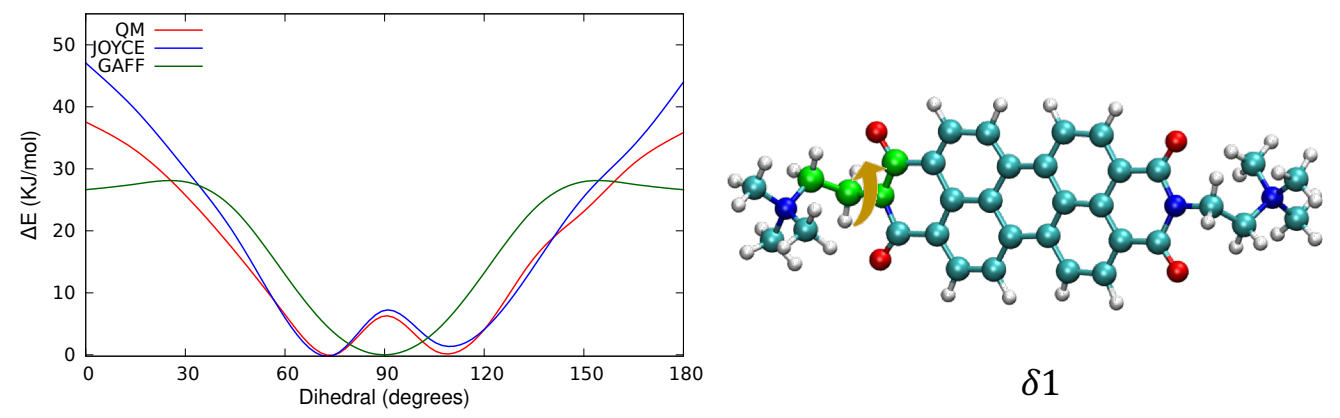

$\delta 1$
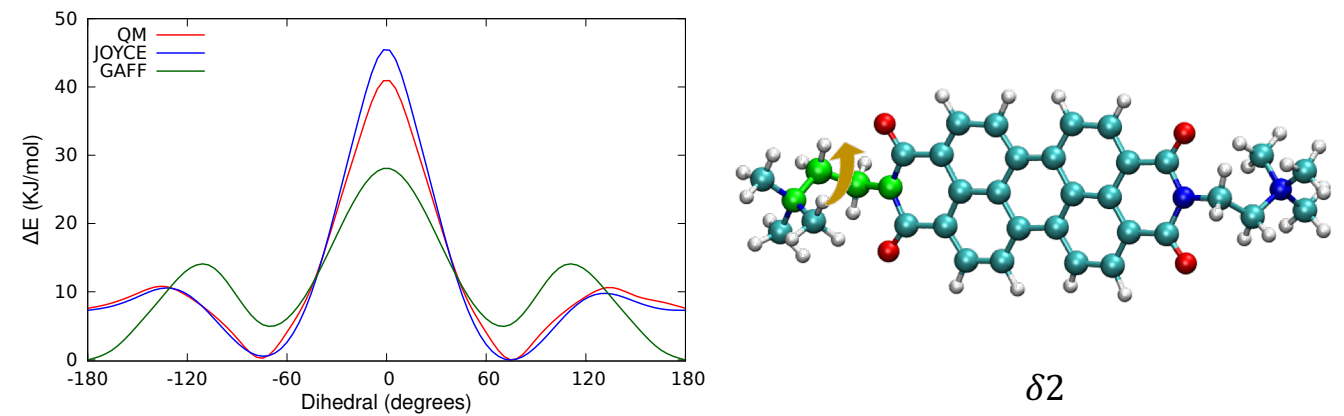

$\delta 2$
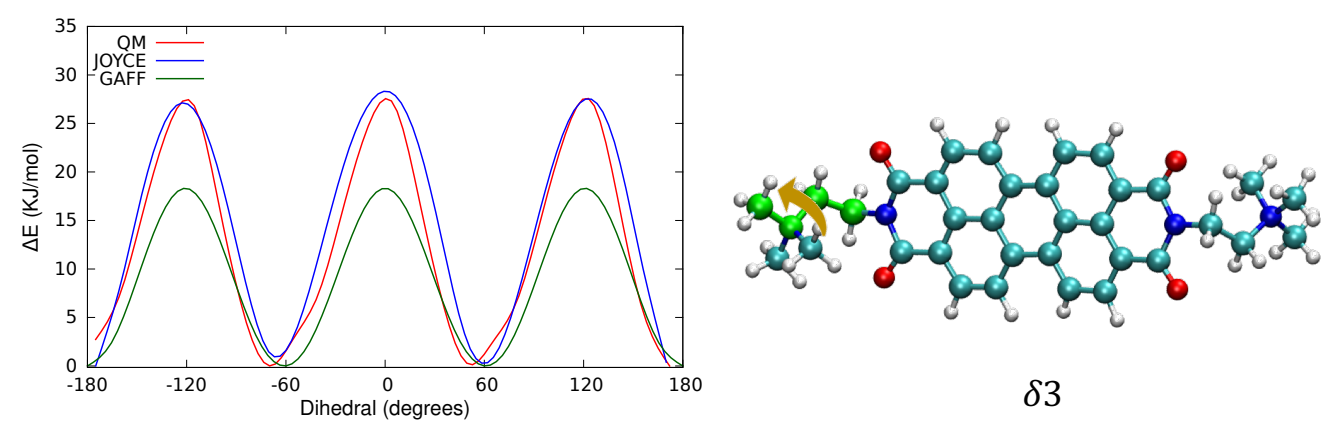

$\delta 3$

Figure 4: Comparison between QM torsional relaxed energy scans (red lines) and the relative MM relaxed profiles computed for the JOYCE (blue) and the GAFF (green) FFs. Each of the monitored PDI's flexible dihedrals is evidenced in the right insets as a orange arrow, and the heavy atom quadruplets used for their definition highlighted in green.

To further gauge the quality of the two considered FFs, we analysed the first 100 ps of the classical MD trajectories in GP, in comparison with the reference AIMD run. The conformational changes, sampled every $0.1 \mathrm{ps}$, have been monitored by considering the most relevant bond lengths of the PDI conjugated core (Table 1) and the flexible chain dihedrals (Figure 5). While the former are important for the accurate prediction of the absorption spectrum, as the $S 0 \rightarrow S 1$ transition is localized on the planar perylene core, ${ }^{16}$ the distribution of the flexible di- 
hedrals along the MD runs is expected to be crucial in dictating the self-aggregation properties. Data in Table 1 show an overall good match between the JOYCE and the DFT distributions, whereas GAFF predicts less accurate bond lengths, especially for the C5-C5 and the N1-C1 bonds (see Figure 3 for label definition), where significant differences around $0.05 \AA$ are found. These discrepancies arise from the transferability requirements of the general purpose nature

\begin{tabular}{l|ccc|c|ccc} 
& DFT & JOYCE & GAFF & & DFT & JOYCE & GAFF \\
\hline N1-C1 & $1.407 \pm 0.029$ & $1.399 \pm 0.031$ & $1.360 \pm 0.024$ & C5-C5 & $1.476 \pm 0.029$ & $1.473 \pm 0.030$ & $1.412 \pm 0.023$ \\
C1-C2 & $1.476 \pm 0.029$ & $1.473 \pm 0.032$ & $1.498 \pm 0.028$ & C2-C6 & $1.417 \pm 0.025$ & $1.413 \pm 0.027$ & $1.393 \pm 0.024$ \\
C2-C3 & $1.379 \pm 0.024$ & $1.377 \pm 0.027$ & $1.392 \pm 0.025$ & C6-C7 & $1.420 \pm 0.026$ & $1.419 \pm 0.030$ & $1.409 \pm 0.023$ \\
C3-C4 & $1.401 \pm 0.026$ & $1.398 \pm 0.026$ & $1.391 \pm 0.025$ & C5-C7 & $1.429 \pm 0.026$ & $1.428 \pm 0.028$ & $1.412 \pm 0.024$ \\
C4-C5 & $1.389 \pm 0.024$ & $1.387 \pm 0.026$ & $1.407 \pm 0.025$ & & & &
\end{tabular}

Table 1: Bond lengths $(\AA)$ distributions and standard deviations computed from the PDI trajectories in vacuo, considering either MD ((GAFF or JOYCE) or AIMD (DFT) runs. The atom labels refer the ones defined for the JOYCE FF, shown in Figure 3

of the GAFF FF, where only three different atom types are used to describe the PDI core: one atom type for $\mathrm{N}$, one for the carbon bearing the oxygen atoms $(\mathrm{C})$ and one for the remaining carbon atoms $\left(\mathrm{C}_{A}\right.$, see Figure $\mathrm{S} 1$ in the Supporting Information). As evident from Table $\mathrm{B}$, this severely limits the capability of GAFF to account for different single (or double) bonds within the perylene core, with the inevitable consequence of the poor description of the bond length alternation with respect to the QM reference. ${ }^{103}$ Conversely, as detailed in the Supporting Information and in Figure 3, a more specific atom type assignment can be adopted in the construction of the QMD-FF, in turn leading to the larger variety of stretching (and bending) parameters emerging from the comparison of the two FFs. As is apparent, despite the simplicity of the model harmonic function, JOYCE QMD-FF is able to reproduce the DFT distributions, indicating a negligible impact of the anharmonic effects.

Figure 5 shows the population distribution of the chain flexible dihedrals $\delta_{1}-\delta_{3}$ obtained with the two FFs and along the reference AIMD trajectory. As suggested from the torsional energy profiles shown in Figure 4, the main lack of the GAFF trajectory in reproducing dihedral dynamics consists in predicting $\delta_{1}$ in a wrong position, centered at $\sim 90^{\circ}$ (instead of $\sim 75^{\circ}$ / $\sim 105^{\circ}$, where the QM minima are located) and delivering for $\delta_{2}$ an asymmetric distribution around $0^{\circ}$. On the contrary, the $\delta_{1}$ and, in particular, $\delta_{2}$ QMD-FF distributions are more closely 

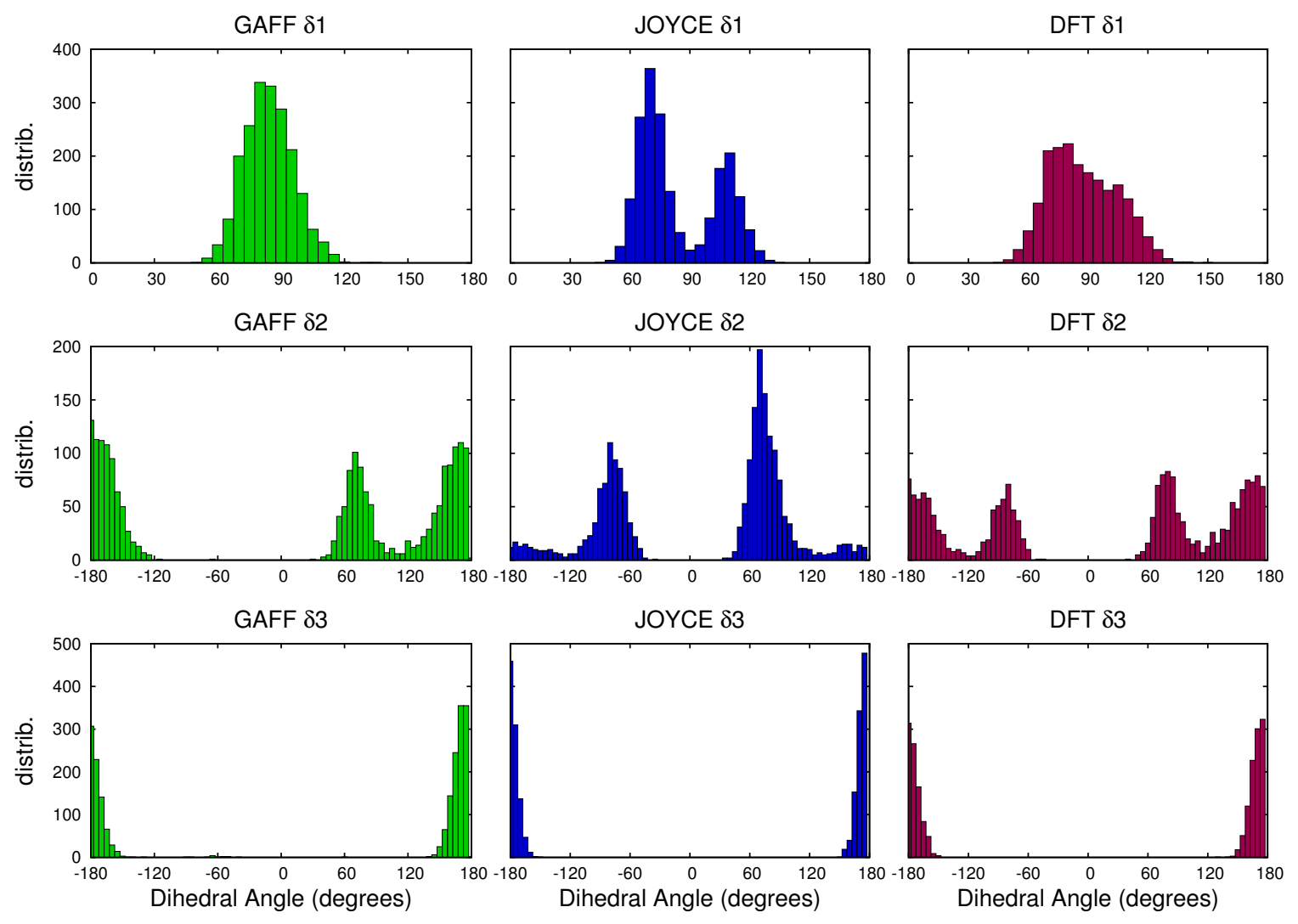

Figure 5: Dihedral distributions computed along the AIMD (DFT) and MD (GAFF, JOYCE) trajectories. The labels of the flexible dihedrals are given in Figure 4.

related to the QM profiles and torsional minima, even if the AIMD simulation seems to indicate lower potential barriers, being the predominance of one peak with respect to the others less pronounced. These differences might arise from the coupling between these torsions, which is neglected in both GAFF and JOYCE FF. Despite such effect has been implemented in the most recent version of the JOYCE procedure, ${ }^{81}$ we chose not to use FF explicit coupling functions, in favour of a more fair comparison between the two tested FFs. Finally, it is worth mentioning that, although this analysis is performed over short MD trajectories, it appears to be sufficient to explore the PDI's PES, as shown in Figures S8-S12 in the Supporting Information, where the data obtained from longer MD simulations ( $\sim 10 \mathrm{~ns})$ do not present notable changes on the overall character of the short-run distributions. 


\subsubsection{Solution}

MD simulations in solution (ACN) revealed even more remarkable differences between GAFF and JOYCE FFs. The comparison between the population distribution of selected internal fast coordinates is reported in Table L and Figures S13-S16 in the Supporting Information and essentially confirm the results obtained in GP, being the stiff modes less sensitive to the solvent. The distributions of the flexible dihedrals are conversely expected to be more effected by the presence of the solvent. In particular, the exchange rate among different minima is usually
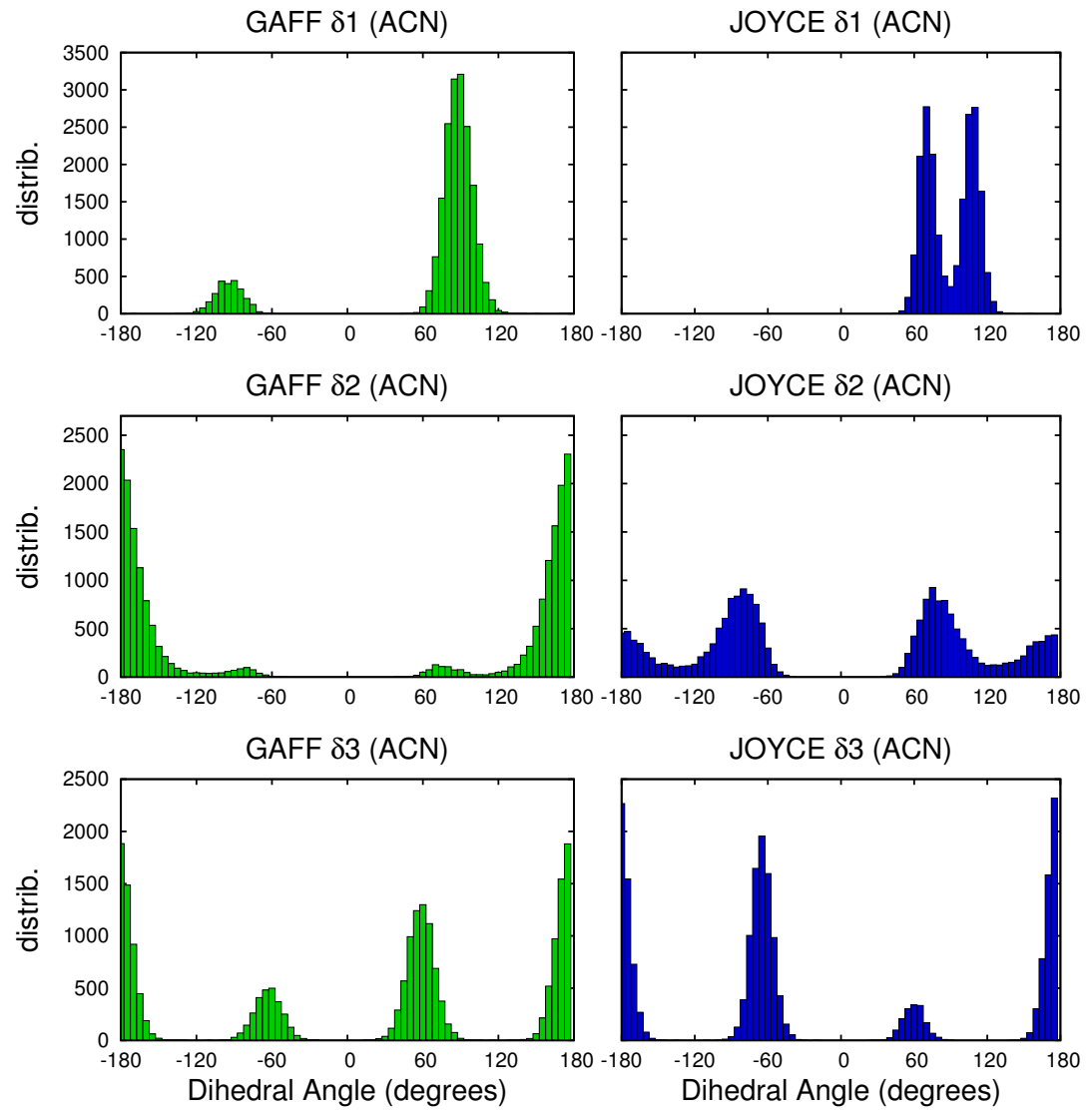

Figure 6: Dihedral distributions computed from the PDI@ACN MD runs by using the GAFF FF and the JOYCE FF. The labels of the flexible dihedrals are given in Figure 4.

higher in solution compared to GP, because the interactions with the solvent might help in overcoming the energy barriers. For this reason, the $\delta_{1}-\delta_{3}$ population distribution, obtained for 
the PDI@ACN system with both FFs, is shown in Figure 6 along the whole $\left[-180^{\circ}-180^{\circ}\right]$ range. As far as $\delta_{1}$ is concerned, JOYCE FF yields two equally intense peaks centered at $\sim 75^{\circ}$ and $\sim 105^{\circ}$, in agreement with two degenerate minima revealed by the QM torsional profiles. On the contrary, as evident in the left top panel, GAFF FF is characterized by two peaks of different intensity, centered at $\pm 90^{\circ}$, meaning that the molecule is visiting the syn conformation, as a consequence of the underestimated syn/anti inter-conversion barrier (see Figure 4). On the same foot, GAFF predicts the local minimum of the $\delta 2$ dihedral at $180^{\circ}$ to be remarkably more overpopulated with respect to the real minimum at $\sim 75^{\circ}$. The conformational analysis presented here, in line with the results obtained in GP, highlights the limitations that could arise from the blind use of a general purpose FF: both the bond length alternation within the perylene core and the flexibility of the side chains are poorly described, possibly delivering inaccuracies in the calculation of the optical and aggregation properties. In the following, we will discuss the first point, that is the accuracy of the absorption spectrum prediction, by using different levels of approximations.

\subsection{Absorption Spectra}

\subsubsection{CEA-VE}

As previously noted, the conformational analysis carried out on the short (100 ps) and long (10 ns) MD runs in GP did not reveal, within the same FF description, any significant differences. For this reason, the short MD runs carried out in vacuo can be confidently used to further assess, with respect to AIMD, the quality of the two FFs concerning the calculations of the optical properties. The three 100 ps trajectories (DFT, GAFF and JOYCE) have been sampled by extracting a configuration every $1 \mathrm{ps,} \mathrm{computing,} \mathrm{to} \mathrm{build} \mathrm{CEA-VE} \mathrm{spectra,} \mathrm{the} \mathrm{vertical}$ transition energy and its intensity. As expected, the good agreement found between JOYCE and DFT descriptions of the PDI core's structure is reflected in the spectra shown in Figure 7. In fact, both the position and the shape of the JOYCE spectrum almost coincide with the ones computed along the DFT trajectory. On the same foot, the discrepancies emerged in the average values and distribution of key internal coordinates, cause GAFF to deliver a significantly $(\sim 0.3 \mathrm{eV})$ redshifted spectrum, with an overestimated intensity, as revealed by Figure S18 in the Supporting 


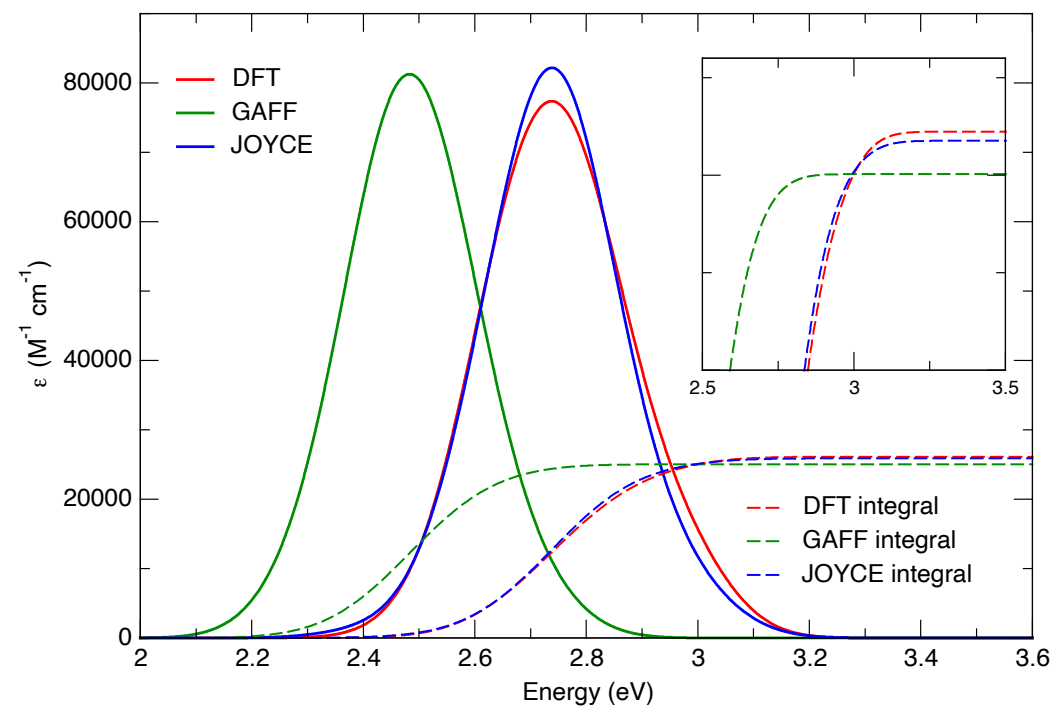

Figure 7: Absorption spectra $\left(\varepsilon(\omega), \mathrm{M}^{-1} \mathrm{~cm}^{-1}\right)$ and integral, computed with the CEA-VE approach along the trajectories of the isolated PDI monomer (GP) obtained with AIMD (DFT, red) or MD (GAFF, green and JOYCE,blue). The vertical transitions have been convoluted by using Gaussian functions having HWHM of 0.05 $\mathrm{eV}$.

Information, where, instead of the molar absorbivity, $\varepsilon(\omega)=C \omega L(\omega)$, the lineshape $L(\omega)$ given in equation (2) is considered.

Turning to the simulations in acetonitrile, as detailed in section 3.3, we tested different schemes to take the solvent into account and Figure 8 collects the resulting CEA-VE spectra. As evident from the first two top panels, when the solvent is accounted for with a given solvation scheme, the GAFF, panel a), and JOYCE, panel b), FFs behave rather similarly with respect to their GP reference spectra. It appears for instance that in both cases the simple EEpc scheme, based on bare point-charges, only predicts a negligible effect, i.e. a slight blue-shift of less than $\sim 0.01 \mathrm{eV}$. Furthermore, when the first solvation layer is included in the QM calculation (QM/EE scheme), both FFs show a more significant red-shift, of $\sim 0.1 \mathrm{eV}$, in the absorption maximum with respect to GP spectra. This is in line with experimental evidences, which indicate, for a series of perylenes, a bathochromic shift of the order of $0.06-0.08 \mathrm{eV}$, increasing 


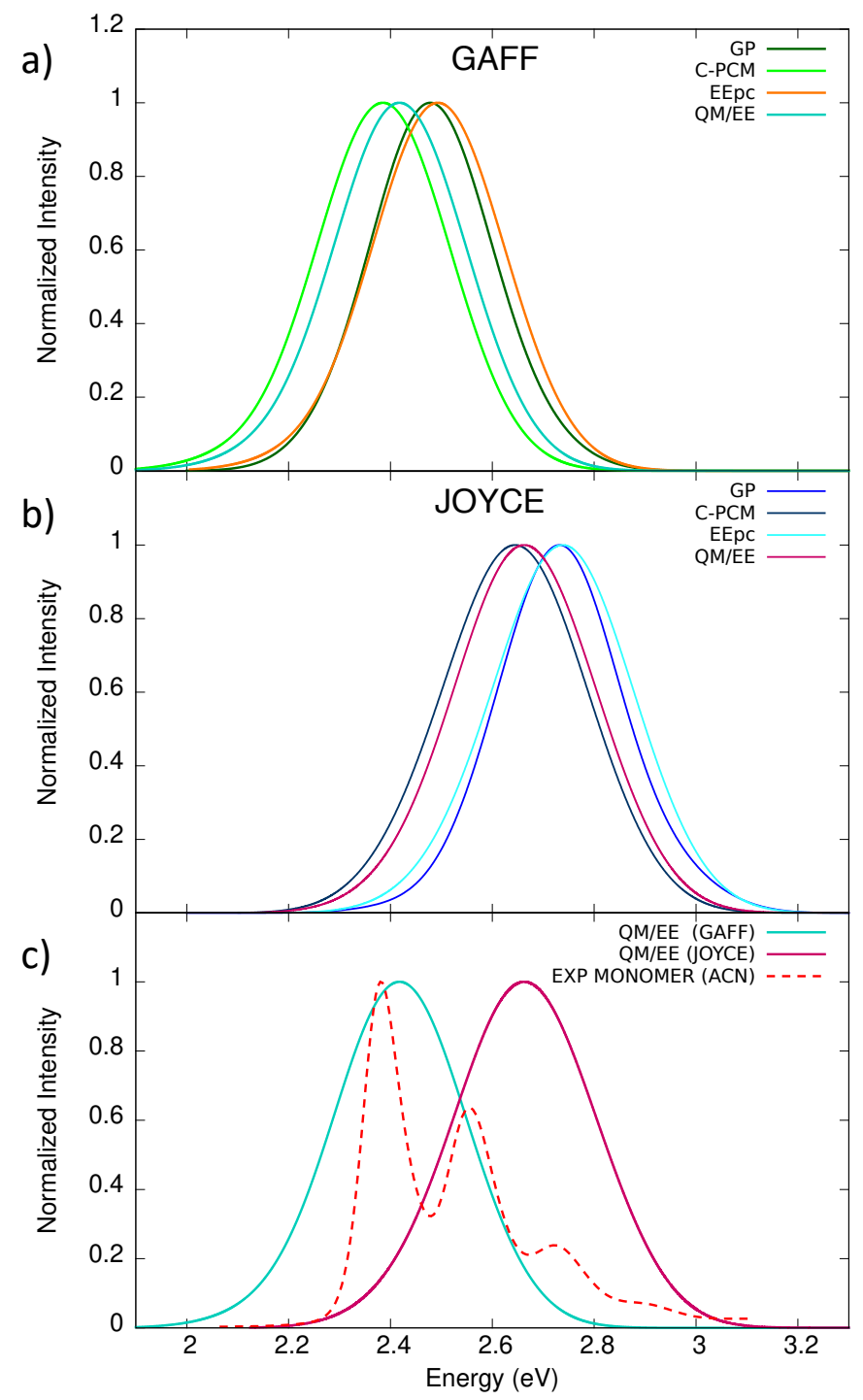

Figure 8: a) CEA-VE spectra computed along the GAFF trajectories: comparison between GP and PDI@ACN, by considering different solvent models (section 3.3); b) the same comparison for CEA-VE spectra computed along the JoYCE trajectories; c) Comparison of the PDI@ACN spectra computed using the QM/EE scheme with the experimental one. ${ }^{10}$ For all the spectra, the vertical transitions have been convoluted by using Gaussian functions having FWHM of $0.1 \mathrm{eV}$.

as the solvent polarity increases. ${ }^{104,105}$ Interestingly, results similar to the QM/EE scheme, can be also obtained with an implicit description of the medium, as in the C-PCM scheme: the main solvent effects seem to be captured, with an overall good agreement with the explicit QM/EE approach obtained at a significantly lower computational cost. It is important to stress, 
however, that implicit solvent schemes can hardly provide a reliable description of PDI selfassembly, nor be used to monitor a possible dynamical solvent response ${ }^{106}$ in the $\pi$ - $\pi$ driven aggregation process. Furthermore, the performances of implicit models for the prediction of solvatochromic shifts in solvents capable of forming hydrogen bonds like ACN would need a careful evaluation. ${ }^{107}$ In these cases, a popular recipe adopts hybrid models where a cluster comprising the solute and few solvent molecules (undergoing specific interactions with the solute) is embedded in PCM. This model is computationally cheap and surely useful, although the lack of the second solvation shell usually induces an overestimation of the solute-solvent interactions. ${ }^{108}$ These cluster models have also been used for vibronic calculations, showing that they may be challenged by large amplitude anharmonic motions of the solvent molecules, possibly overestimated by the lack of the second shell molecules. ${ }^{54} \mathrm{Ad}-\mathrm{MD} \mid \mathrm{gVH}$ method solves this issue at the cost to describe solvent motions classically. ${ }^{56}$

In the bottom panel of Figure 8, we eventually compare the CEA-VE spectra computed with the most refined model (QM/EE), with the one experimentally recorded in ACN. ${ }^{10}$ While the JOYCE-based spectrum is appreciably blue-shifted, $(0.28 \mathrm{eV}$ or $0.21 \mathrm{eV}$ considering either the maximum or the first moment, as recommended in ref. ${ }^{109}$ ), an almost perfect agreement with experiment is obtained by using the GAFF trajectory (first moment: $-0.08 \mathrm{eV}$, maximum: +0.04 $\mathrm{eV}$ ). Based on the results of both the conformational analysis and the performances of the GP spectra with respect to the AIMD reference, this result is rather surprising and documents a fortuitous error cancellation, similarly to what recently reported for a metallo-organic dye complex. ${ }^{110}$ On the one hand, the $\sim 0.3 \mathrm{eV}$ red-shift found in GAFF-based spectra of the isolated PDI with respect to reference AIMD, evident in Figure 7, is due to the inaccurate bond lengths and angles of the perylene core, yielded by the GAFF simulations. Given the scarce flexibility of the $\pi$-core, it can be therefore expected that the spectra in solution will be affected by such a bias in a rather similar way. On the other hand, in the calculation of the excitation energy a blueshift of about the same magnitude was reported for the CAM-B3LYP functional with respect to the experiment. ${ }^{16}$ In summary, even if accounting for vibronic effects actually sensibly reduces the CAM-B3LYP deficiency (see next section), at CEA-VE level its combined effect with the error due to the geometrical inaccuracies leads, in the case of the GAFF, to the fortuitous very 
good agreement appearing in Figure 8. Nonetheless, the most important conclusion that can be drawn from the bottom panel, is the confirmation that, regardless of the employed FF, the CEAVE approach is not able to reproduce the PDI vibronic progressions, which instead requires to take into account the coupling between electronic and vibrational quantum states explicitly, as discussed in the next section.

\subsubsection{MQC spectra}

Figure 9a displays the $A d-M D \mid g V H$ spectra calculated by averaging over the 100 snapshots extracted from the DFT, GAFF and JOYCE MD trajectories in GP (100 ps runs). All the individual vibronic spectra, computed at each frame, are displayed in Figures S21-S23 of the Supporting Information. Both GAFF and JOYCE predictions are very close to the reference DFT spectrum: a quite remarkable result because, as evidenced in Figure 7, at CEA-VE level the GAFF spectrum is off (red-shifted) by $\sim 0.3 \mathrm{eV}$, due to the wrong sampling of the distribution of the stiff coordinates. This finding shows that $A d-M D \mid g V H$ makes a very good job in recovering such an error. This is possible because, at each snapshot, the $\mathrm{gVH}$ method reconstructs harmonic PES along the stiff coordinates, by extrapolating the equilibrium geometry from the gradient and Hessian. Since stiff coordinates have short-amplitude oscillations, this procedure reveals to be quite accurate even starting from significantly displaced geometries, like those sampled by GAFF. Results are expected to be different for systems with larger anharmonicites and couplings between soft and stiff coordinates. A more detailed analysis shows that the GAFF peaks maxima are slightly blue-shifted, while JOYCE's ones slightly red-shifted, but closer to the reference. The intensity of the JOYCE spectrum is only apparently slightly smaller. Actually, the integrated intensity of JOYCE spectrum is extremely similar to the AIMD reference, indicating that the slightly smaller maxima are compensated by a slightly broader spectrum. JOYCE vibronic spectrum in fact becomes more intense than the DFT one at energies $\sim>3.1 \mathrm{eV}$. At variance, as shown in Figure 7, the total intensity of GAFF spectrum is truly, though very slightly, higher. On the balance, however, the vibronic peaks are quite well resolved along all three trajectories, they have a very regular shape and a quite similar width. The fact that vibronic resolution is preserved after an average over 100 snapshots indicates that no spurious broadening 


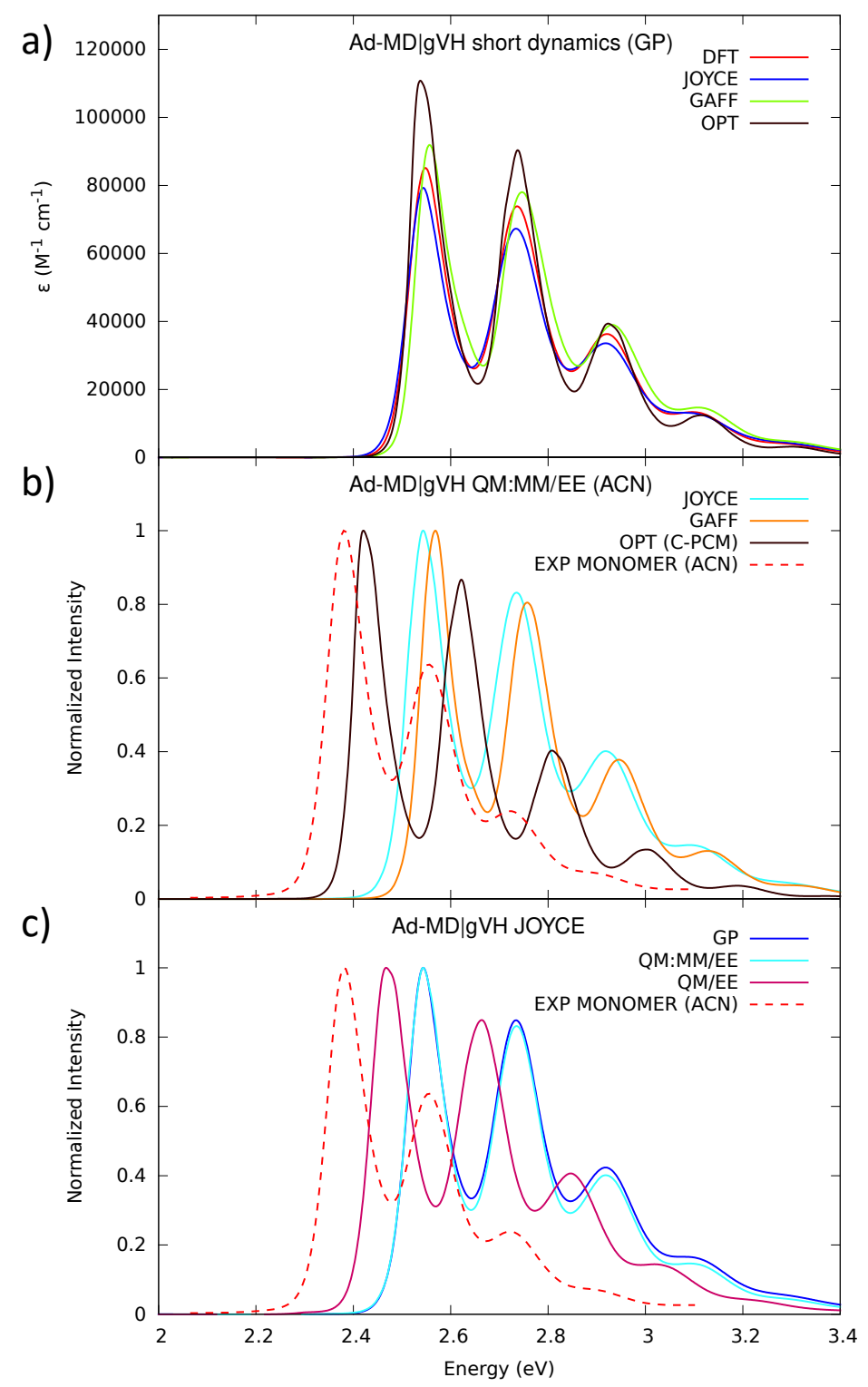

Figure 9: a) $A d-M D \mid g V H$ spectra computed in GP along the AIMD and MD trajectories. The simple vibronic spectrum (OPT) computed for the single optimized PDI is also reported for comparison; b) Comparison among GAFF and JOYCE based $A d-M D \mid g V H$ spectra computed for the PDI@ACN system with the QM:MM/EE scheme, the OPT spectrum in PCM, and the experimental one; c) Effect of the solvent model scheme on the $A d-M D \mid g V H$ PDI spectra in ACN computed along the JOYCE trajectory. The experimental spectrum ${ }^{10}$ is also reported for comparison. In all panels, the single vibronic spectra concurring in the $A d-M D \mid g V H$ lineshape were calculated by including the torsions of the alkyl chains in the classical set, and considering an FWHM of $0.01 \mathrm{eV}$. 
mechanism due to excessive fluctuations of the molecular structures occur either during classical MD runs. This is especially noteworthy in the QMD-FF case, considering that JOYCE FF was built over a much smaller set of internal coordinates with respect to GAFF, avoiding for instance the burden connected in the inclusion of all nonbonded intramolecular distances, as discussed in more detail in the Supporting Information. Finally, it should be noted that in the $A d-M D \mid g V H$ spectra shown in Figure 9, all vibrational DoF were treated at quantum level, except the $\delta_{1}-\delta_{3}$ torsions of the flexible lateral chains and the rotations of the methyl groups, which were included in the classical set. In Figure S19 of the Supporting information we show that treating all independent dihedrals of the lateral chains (i.e. including also pyramidalizations and out-of-plane motions) as classical coordinates leads to only marginally different spectra. It is also interesting to compare the predictions of $\mathrm{Ad}-\mathrm{MD} \mid \mathrm{gVH}$ with a standard static vibronic $\mathrm{VH}$ approach, in which the spectrum is computed assuming harmonic approximation for all DoFs. Panel a) in Figure 9 displays such spectrum (OPT, black line), and shows that it is indeed quite similar to the $A d-M D \mid g V H$ ones, both in its position and overall shape. However the static vibronic spectrum is more resolved and the bands are slightly narrower. The same behaviour is observed considering the average of Boltzmann static spectra at all the local minima along the flexible torsions (Figure S20 in the Supporting Information). This finding suggests that fluctuations of the lateral chains do induce a broadening, captured by the $A d-M D \mid g V H$ methodology, but this is minimal, in agreement with the scarce dependence of the spectra on the nature of the side chains argued in refs. $[14,16]$. This also explains why the low-energy peaks of the static spectrum appear higher, although the total intensity of the spectra is practically identical.

Figure $9 \mathrm{~b}$ plots the $A d-M D \mid g V H$ spectra computed in ACN along the GAFF or JOYCE trajectories using the QM:MM/EE solvent scheme, and compares them with the experiment. ${ }^{10}$ In this case, beside the torsions of the lateral perylene chains also all solvent DOFs were included in the classical set. The figure also reports the results of a static vibronic computation with the VH method, obtained describing solvent effects with C-PCM (OPT). $A d-M D \mid g V H$ spectral shapes are in good agreement with experiments, apart from an overestimation of the vibronic progression, i.e. of the relative intensity of the excited bands with respect to the lowestenergy $(0 \rightarrow 0)$ one. Such an overestimation is even slightly larger with C-PCM and can be 
thus ascribed to the use of CAM-B3LYP. As observed in GP, also in the PDI@ACN system $A d-M D \mid g V H$ delivers spectra of rather similar quality, when applied to either GAFF or JOYCE trajectories. Focusing on the spectral position with respect to the experiment, MQC spectra are blue-shifted by $\sim 0.2 \mathrm{eV}$ in combination with JOYCE and slightly more with GAFF. Moreover, comparison of Figures 9a (GP) and 9b (ACN) shows that describing the solvent at QM:MM/EE level does not significantly affect either the spectral shape or the position. The situation is different when the static OPT approach is employed. C-PCM clearly does not describe the molecularity of the solvent, but it accounts for mutual solute/solvent polarizations which, on the other hand, are neglected in the QM:MM/EE model. The OPT/C-PCM scheme (in nonequilibrium regime, as recommended in ref. ${ }^{62}$ ) indeed predicts a remarkable solvent red-shift of $\sim 0.11 \mathrm{eV}$ in line with experiments. Despite the improved agreement with the experimental peaks position, the reliability of (linear response) C-PCM in accounting for the specific solvent shift in ACN should be taken with care, considering the failure of the same model in reproducing the experimental shift ${ }^{104,105}$ between ACN and diethyl ether, as displayed in Figure S24.

Finally, it is worth noticing that the prediction of OPT/C-PCM is strongly dependent on the adopted solvation regime: Figure S25 in the Supporting Information shows that switching from the non-equilibrium regime (usually adopted for vertical transitions but quite slower for vibronic spectra, due to the numerical calculation of the Hessians implemented in GAUSSIAN16) to the equilibrium regime (the default choice for optimizations and Hessians and much faster) worsens the overestimation of the vibronic progressions and the position of the spectrum, remarkably increasing the shift with respect to GP to $\sim 0.35 \mathrm{eV}$.

In order to incorporate in the $A d-M D \mid g V H$ spectra the effect of the mutual solute/solvent polarization, accounted in average by C-PCM, we repeated the calculations along the JOYCE trajectory adopting the more refined QM/EE model scheme, as defined for the vibronic calculations in section 3.3. Figure 9c shows that the mutual polarization of solute and solvent molecules red-shifts the spectrum, by $\sim 0.08 \mathrm{eV}$, approximately halving the error with respect to the experiment. It is noteworthy that the adopted procedure does not describe the effect of the solvent/solute polarization on the spectral shape: in order to do it within the $A d-M D \mid g V H$ scheme, it would be necessary to recompute with a QM/EE protocol not only the energies but 
also the gradients and Hessians of the initial and final states at each snapshot. This would be extremely costly and is beyond the aims of the present work.

\section{Conclusions}

An original MQC procedure, ${ }^{56}$ recently proposed by us to tackle absorption spectra of flexible dyes in condensed phase, is here applied to a PDI dye in acetonitrile solution. The $A d-$ $M D \mid g V H$ procedure yielded an electronic absorption spectrum which correctly exhibited a pronounced vibronic structure, within a good accuracy with respect to the experimental spectrum, ${ }^{10}$ thus overcoming the intrinsic inability of the standard CEA-VE approach to handle vibronic features. Considering the most refined procedure adopted, we eventually obtained quite accurate results, with an error on the position of the vibrationally resolved spectrum around $0.1 \mathrm{eV}$, which was ascribed to the employed CAM-B3LYP functional, also responsible for the overestimation of the vibronic progressions. Therefore, here we show that $A d-M D \mid g V H$ is a solid methodology for forthcoming investigations of the spectroscopy of PDI aggregates.

On more general grounds, the different schemes and options explored during this computational study, allowed us to draw a number of conclusions, whose interest goes beyond the application to the PDI species. First, as far as the FF's accuracy is concerned, GAFF and JOYCE FFs lead to remarkably different samplings, and this is reflected in the CEA-VE spectra computed along the two trajectories. In the gas phase, at difference with JOYCE, whose spectrum coincides with that computed along the AIMD trajectory used for reference, GAFF is red-shifted by $0.3-0.4 \mathrm{eV}$. The reason can be traced back to an intrinsic inconsistency in the application of CEA-VE methodology in combination with MD driven by a general purpose FF. CEA-VE is in fact based on the application of the classical FC principle. According to it, one should select a level of theory, sample the minimum region in the ground state and then compute the vertical transitions. This is what is done with the AIMD carried out with the CAM-B3LYP functional, and with the JOYCE FF, built to minimize the differences with the DFT PES. On the contrary, GAFF describes a different PES, consequently inducing the MD runs to explore a configurational space that does not correspond to the minimum region of the DFT potential. Nonetheless, when the solvent is introduced, the GAFF spectrum at CEA-VE level seems to be 
in better agreement with the experimental one. We have clearly shown that this is the effect of a fortuitous compensation of errors. On one side a wrong sampling, on the other side the fact that vibronic contributions are neglected and CAM-B3LYP overestimates the transition energies. ${ }^{16}$

Turning to MQC spectra, in gas phase $A d-M D \mid g V H$ delivers similar results with all three samplings, thus correcting the inherent errors found for GAFF. Beside being another important advantage of our procedure, this finding is quite instructive, because it unveils that for PDI the errors of GAFF sampling more relevant to the spectrum concern stiff modes. This is the reason why they can be corrected by the quadratic extrapolation performed by $g V H$ to locate the minima of the initial and final PES along the stiff coordinates. When the solvent is introduced, if a not-polarizable point-charge embedding model is considered, interestingly, none of the trajectories is able to produce a spectrum with the expected solvent shift with respect to the gas phase. In fact, we demonstrated that mutual solute-solvent polarization is indeed important to reproduce the solvent shift, and it was introduced in our procedure by considering a first QM solvation shell. In this particular case, our calculations indicate that these effects could also be inserted with specific settings of the C-PCM, but we do expect this will not be the case for $\pi$-aggregates, where the molecularity of the solvent will be important and PCM cavities may be unphysically large. As an additional general observation, it is also worth highlighting that our results document that accounting for the quantum nature of the stiff modes does not simply introduce vibronic resolution: in fact, once the vibronic effects are taken into account, a net redshift of the spectrum maximum, also described in literature, ${ }^{109}$ is predicted by $\sim 0.2 \mathrm{eV}$, both in gas and in ACN, strongly improving the agreement with experiment. Many recent works have highlighted the necessity to adopt mixed approaches to introduce solvent effects on electronic spectra, based on multiscale QM:MM partitions. In this way, the effect of the extension of the QM partition on the description of solvent shifts, ${ }^{111}$ solvent broadening, ${ }^{75}$ or other photochemical properties, ${ }^{112}$ has been investigated. From the methodological point of view, beside the possibility of combining explicit and continuum models, ${ }^{113}$ recent progresses have been made in the use of ab initio dynamics with non periodic boundary conditions, ${ }^{114}$ polarizable force fields, ${ }^{115,116}$ or clustering methods combined to the perturbation matrix method to recover the effect of local fluctuations of the environment. ${ }^{51,52}$ Interestingly, most of the proposed compu- 
tational protocols are often indicated as MQC, with implicit reference to the description of the electrons, or more precisely, to whether solute/solvent interactions are computed according to quantum mechanics or with force fields. ${ }^{115,116}$ Here on the contrary, as done also in refs. 71,85, we mainly focused on a different aspect of the problem, i.e the need for a MQC description of the nuclear motion. These two research topics are clearly related and it is easy to foresee that in the next years the different developments in the two fields will be combined in innovative ways.

Finally, focusing again on the present application, the similarity of the $A d-M D \mid g V H$ spectra obtained from the GAFF and JOYCE based trajectories, clearly confirms that a reliable description of the lateral pendants is not really required for an accurate spectrum of the PDI monomer, considering the significant errors in the flexible lateral chain GAFF populations, revealed by our conformational analysis. This is further corroborated by the comparison with full quantum static vibronic calculation that treats also the soft coordinates as harmonic quantum modes: the good agreement with the $A d-M D \mid g V H$ spectrum shows that they just induce a very limited broadening. Yet, even if in the specific case the flexibility of the lateral chains has a mild effect on the spectrum, their reliable description is expected to have a larger impact on the spectra of aggregates. On this respect the fact that JOYCE FF is able to properly reproduce both the oscillations of stiff modes and the flexibility of the soft ones is very promising: the extension of the $A d-M D \mid g V H$ procedure in combination with JOYCE to the study of aggregates is currently in progress. The major step is to substitute the engine for the computation of vibronic spectra in case of negligible inter-state couplings $(\mathrm{gVH})$ with a model that explicitly account for such couplings like Linear vibronic coupling (LVC) models ${ }^{117,118}$ and exciton+CT models.

\section{Supporting Information}

Details on the FF parametrization including a complete list of FFs parameters, analysis of the MD trajectories and atomic radial pair functions calculated between the PDI and the solvent molecules. Absorption lineshape and integrals computed from the PDI MD runs in GP. Calculated $A d-M D \mid g V H$ spectra considering all chain dihedrals as classical coordinates. Comparison between the $A d-M D \mid g V H$ spectra and the average of Boltzmann static spectra. Effects 
on fully quantum spectra due to PCM models (equilibrium and non-equilibrium) and PCM solvents (ACN and ether). Vibronic spectra computed for each individual snapshot along the PDI MD trajectories.

\section{Acknowledgements}

MP thanks ANR JCJC HELIOSH2 (ANR-17-CE05-0007-01) and the COMETE project (COnception in silico de Matériaux pour l'EnvironnemenT et l'Energie) co-funded by the European Union under the program 'FEDER-FSE Lorraine et Massif des Vosges 2014-2020 for financial support. HPC resources from the mésocentre EXPLOR of Université de Lorraine (Project 2018CPMXX0602) and LPCT local computational resources are also acknowledged. 


\section{References}

[1] Zhang, Z.-G.; Qi, B.; Jin, Z.; Chi, D.; Qi, Z.; Li, Y.; Wang, J. Perylene diimides: a thickness-insensitive cathode interlayer for high performance polymer solar cells Energy Environ. Sci. 2014, 7, 1966-1973.

[2] Kozma, E.; Catellani, M. Perylene diimides based materials for organic solar cells Dyes Pigm. 2013, 98, 160 - 179.

[3] Zhang, B.; Lyskov, I.; Wilson, L. J.; Sabatini, R. P.; Manian, A.; Soleimaninejad, H.; White, J. M.; Smith, T. A.; Lakhwani, G.; Jones, D. J.; Ghiggino, K. P.; Russo, S. P.; Wong, W. W. H. FRET-enhanced photoluminescence of perylene diimides by combining molecular aggregation and insulation J. Mater. Chem. C 2020, 8, 8953-8961.

[4] Dirian, K.; Bauroth, S.; Roth, A.; Syrgiannis, Z.; Rigodanza, F.; Burian, M.; Amenitsch, H.; Sharapa, D. I.; Prato, M.; Clark, T.; Guldi, D. M. A water-soluble, bay-functionalized perylenediimide derivative - correlating aggregation and excited state dynamics Nanoscale 2018, 10, 2317-2326.

[5] Krieg, E.; Niazov-Elkan, A.; Cohen, E.; Tsarfati, Y.; Rybtchinski, B. Noncovalent Aqua Materials Based on Perylene Diimides Acc. Chem. Res. 2019, 52, 2634-2646;

[6] Yang, Z.; Chen, X. Semiconducting Perylene Diimide Nanostructure: Multifunctional Phototheranostic Nanoplatform Acc. Chem. Res. 2019, 52, 1245-1254.

[7] Obaidulla, S. M.; Habib, M. R.; Khan, Y.; Kong, Y.; Liang, T.; Xu, M. MoS2 and Perylene Derivative Based Type-II Heterostructure: Bandgap Engineering and Giant Photoluminescence Enhancement Adv. Mater. Interfaces 2020, 7, 1901197.

[8] Ronconi, F.; Syrgiannis, Z.; Bonasera, A.; Prato, M.; Argazzi, R.; Caramori, S.; Cristino, V.; Bignozzi, C. A. Modification of Nanocrystalline WO3 with a Dicationic Perylene Bisimide: Applications to Molecular Level Solar Water Splitting J. Am. Chem. Soc. 2015, 137, 4630-4633. 
[9] Bonchio, M.; Syrgiannis, Z.; Burian, M.; Marino, N.; Pizzolato, E.; Dirian, K.; Rigodanza, F.; Volpato, G. A.; La Ganga, G.; Demitri, N.; et al. Hierarchical organization of perylene bisimides and polyoxometalates for photo-assisted water oxidation Nat. Chem. 2019, 11, 146-153.

[10] Berardi, S.; Cristino, V.; Canton, M.; Boaretto, R.; Argazzi, R.; Benazzi, E.; Ganzer, L.; Borrego Varillas, R.; Cerullo, G.; Syrgiannis, Z.; Rigodanza, F.; Prato, M.; Bignozzi, C. A.; Caramori, S. Perylene Diimide Aggregates on Sb-Doped $\mathrm{SnO}_{2}$ : Charge Transfer Dynamics Relevant to Solar Fuel Generation J. Phys. Chem. C 2017, 121, 17737-17745.

[11] Hestand, N. J.; Spano, F. C. Expanded theory of H-and J-molecular aggregates: the effects of vibronic coupling and intermolecular charge transfer Chem. Rev. 2018, 118, 7069-7163.

[12] Ahrens, M. J.; Sinks, L. E.; Rybtchinski, B.; Liu, W.; Jones, B. A.; Giaimo, J. M.; Gusev, A. V.; Goshe, A. J.; Tiede, D. M.; Wasielewski, M. R. Self-assembly of supramolecular light-harvesting arrays from covalent multi-chromophore perylene-3, 4: 9, 10-bis (dicarboximide) building blocks J. Am. Chem. Soc. 2004, 126, 8284-8294.

[13] Wang, W.; Han, J. J.; Wang, L.-Q.; Li, L.-S.; Shaw, W. J.; Li, A. D. Dynamic $\pi$ - $\pi$ stacked molecular assemblies emit from green to red colors Nano Lett. 2003, 3, 455-458.

[14] Würthner, F. Perylene bisimide dyes as versatile building blocks for functional supramolecular architectures Chem. Commun. 2004, 1564-1579.

[15] Balakrishnan, K.; Datar, A.; Naddo, T.; Huang, J.; Oitker, R.; Yen, M.; Zhao, J.; Zang, L. Effect of side-chain substituents on self-assembly of perylene diimide molecules: morphology control J. Am. Chem. Soc. 2006, 128, 7390-7398.

[16] Segalina, A.; Assfeld, X.; Monari, A.; Pastore, M. Computational Modeling of Exciton Localization in Self-Assembled Perylene Helices: Effects of Thermal Motion and Aggregate Size J. Phys. Chem. C 2019, 123, 6427-6437. 
[17] Zhao, H.; Zhang, Y.-y.; Xu, H.; He, Z.-m.; Zhang, Z.-L.; Zhang, H.-q. Synthesis and properties of perylene diimide dyes bearing unsymmetrical and symmetrical phenoxy substituents at bay positions Tetrahedron 2015, 71, 7752-7757.

[18] Clark, A. E.; Qin, C.; Li, A. D. Beyond exciton theory: a time-dependent DFT and Franck- Condon study of perylene diimide and its chromophoric dimer J. Am. Chem. Soc. 2007, 129, 7586-7595.

[19] Houari, Y.; Laurent, A. D.; Jacquemin, D. Spectral signatures of perylene diimide derivatives: insights from theory J. Phys. Chem. C 2013, 117, 21682-21691.

[20] Kennehan, E. R.; Grieco, C.; Brigeman, A. N.; Doucette, G. S.; Rimshaw, A.; Bisgaier, K.; Giebink, N. C.; Asbury, J. B. Using molecular vibrations to probe exciton delocalization in films of perylene diimides with ultrafast mid-IR spectroscopy Phys. Chem. Chem. Phys. 2017, 19, 24829-24839.

[21] Zhao, H.-M.; Pfister, J.; Settels, V.; Renz, M.; Kaupp, M.; Dehm, V. C.; Würthner, F.; Fink, R. F.; Engels, B. Understanding ground-and excited-state properties of perylene tetracarboxylic acid bisimide crystals by means of quantum chemical computations $J$. Am. Chem. Soc. 2009, 131, 15660-15668.

[22] Chen, Z.; Stepanenko, V.; Dehm, V.; Prins, P.; Siebbeles, L. D.; Seibt, J.; Marquetand, P.; Engel, V.; Würthner, F. Photoluminescence and conductivity of self-assembled $\pi-\pi$ stacks of perylene bisimide dyes Chem. Eur. J. 2007, 13, 436-449.

[23] Würthner, F.; Chen, Z.; Dehm, V.; Stepanenko, V. One-dimensional luminescent nanoaggregates of perylene bisimides Chem. Commun. 2006, 1188-1190.

[24] Spano, F. C.; Meskers, S. C. J.; Hennebicq, E.; Beljonne, D. Probing Excitation Delocalization in Supramolecular Chiral Stacks by Means of Circularly Polarized Light: Experiment and Modeling J. Am. Chem. Soc. 2007, 129, 7044-7054.

[25] Settels, V.; Schubert, A.; Tafipolski, M.; Liu, W.; Stehr, V.; Topczak, A. K.; Pflaum, J.; Deibel, C.; Fink, R. F.; Engel, V.; et al. Identification of ultrafast relaxation processes as a 
major reason for inefficient exciton diffusion in perylene-based organic semiconductors J. Am. Chem. Soc. 2014, 136, 9327-9337.

[26] Fulton, R. L.; Gouterman, M. Vibronic coupling. I. Mathematical treatment for two electronic states J. Chem. Phys. 1961, 35, 1059-1071.

[27] Fulton, R. L.; Gouterman, M. Vibronic coupling. II. Spectra of dimers J. Chem. Phys. 1964, 41, 2280-2286.

[28] Hemenger, R. A theory of optical absorption by aggregates of large molecules $J$. Chem. Phys. 1977, 66, 1795-1801.

[29] Knapp, E.; Scherer, P.; Fischer, S. On the lineshapes of vibronically resolved molecular aggregate spectra. application to pseudoisocyanin (PIC) Chem. Phys. Lett. 1984, 111, 481-486.

[30] Spano, F. C. The spectral signatures of Frenkel polarons in H-and J-aggregates Acc. Chem. Res. 2010, 43, 429-439.

[31] Pochas, C. M.; Kistler, K. A.; Yamagata, H.; Matsika, S.; Spano, F. C. Contrasting photophysical properties of star-shaped vs linear perylene diimide complexes J. Am. Chem. Soc. 2013, 135, 3056-3066.

[32] Eisfeld, A.; Briggs, J. The J-and H-bands of organic dye aggregates Chem. Phys. 2006, 324, 376-384.

[33] Schröter, M.; Ivanov, S. D.; Schulze, J.; Polyutov, S. P.; Yan, Y.; Pullerits, T.; Kühn, O. Exciton-vibrational coupling in the dynamics and spectroscopy of Frenkel excitons in molecular aggregates Phys. Rep. 2015, 567, 1-78.

[34] Seibt, J.; Marquetand, P.; Engel, V.; Chen, Z.; Dehm, V.; Würthner, F. On the geometry dependence of molecular dimer spectra with an application to aggregates of perylene bisimide Chem. Phys. 2006, 328, 354-362. 
[35] Seibt, J.; Winkler, T.; Renziehausen, K.; Dehm, V.; Würthner, F.; Meyer, H.-D.; Engel, V. Vibronic transitions and quantum dynamics in molecular oligomers: A theoretical analysis with an application to aggregates of perylene bisimides J. Phys. Chem. A 2009, $113,13475-13482$.

[36] Spano, F. C.; Yamagata, H. Vibronic coupling in J-aggregates and beyond: a direct means of determining the exciton coherence length from the photoluminescence spectrum $J$. Phys. Chem. B 2011, 115, 5133-5143.

[37] Ambrosek, D.; Kohn, A.; Schulze, J.; Kühn, O. Quantum chemical parametrization and spectroscopic characterization of the frenkel exciton hamiltonian for a J-aggregate forming perylene bisimide dye J. Phys. Chem. A 2012, 116, 11451-11458.

[38] Ritschel, G.; Suess, D.; Möbius, S.; Strunz, W. T.; Eisfeld, A. Non-Markovian Quantum State Diffusion for temperature-dependent linear spectra of light harvesting aggregates $J$. Chem. Phys. 2015, 142, 034115.

[39] Clark, A. E.; Qin, C.; Li, A. D. Q. Beyond Exciton Theory: A Time-Dependent DFT and Franck-Condon Study of Perylene Diimide and Its Chromophoric Dimer J. Am. Chem. Soc. 2007, 129, 7586-7595.

[40] Oltean, M.; Calborean, A.; Mile, G.; Vidrighin, M.; Iosin, M.; Leopold, L.; Maniu, D.; Leopold, N.; Chiş, V. Absorption spectra of PTCDI: A combined UV-Vis and TD-DFT study Spectrochim. Acta A 2012, 97, 703 - 710.

[41] Pan, F.; Gao, F.; Liang, W.; Zhao, Y. Nature of Low-Lying Excited States in HAggregated Perylene Bisimide Dyes: Results of TD-LRC-DFT and the Mixed Exciton Model J. Phys. Chem. B 2009, 113, 14581-14587.

[42] Mukazhanova, A.; Trerayapiwat, K. J.; Mazaheripour, A.; Wardrip, A. G.; Frey, N. C.; Nguyen, H.; Gorodetsky, A. A.; Sharifzadeh, S. Accurate First-Principles Calculation of the Vibronic Spectrum of Stacked Perylene Tetracarboxylic Acid Diimides J. Phys. Chem. A 2020, 124, 3055-3063. 
[43] Padula, D.; Santoro, F.; Pescitelli, G. A simple dimeric model accounts for the vibronic ECD spectra of chiral polythiophenes in their aggregated states $R S C A d v$. 2016, 6, 3793837943.

[44] Prampolini, G.; Livotto, P. R.; Cacelli, I. Accuracy of Quantum Mechanically Derived Force-Fields Parameterized from Dispersion-Corrected DFT Data: The Benzene Dimer as a Prototype for Aromatic Interactions J. Chem. Theory Comput. 2015, 11, 5182-5196.

[45] Kraner, S.; Prampolini, G.; Cuniberti, G. Exciton Binding Energy in Molecular Triads $J$. Phys. Chem. C 2017, 121, 17088-17095.

[46] Greff da Silveira, L.; Jacobs, M.; Prampolini, G.; Livotto, P. R.; Cacelli, I. Development and Validation of Quantum Mechanically Derived Force-Fields: Thermodynamic, Structural, and Vibrational Properties of Aromatic Heterocycles J. Chem. Theory Comput. 2018, 14, 4884-4900.

[47] Belletti, G.; Schulte, E.; Colombo, E.; Schmickler, W.; Quaino, P. Development of force fields for binary systems: Application to a dimethylsulfoxide (DMSO) - Oxygen mixture Chem. Phys. Lett. 2019, 735, 136778.

[48] Claridge, K.; Troisi, A. Developing Consistent Molecular Dynamics Force Fields for Biological Chromophores via Force Matching J. Phys. Chem. B 2019, 123, 428-438.

[49] Jiang, L.; Rogers, D. M.; Hirst, J. D.; Do, H. Force Fields for Macromolecular Assemblies Containing Diketopyrrolopyrrole and Thiophene J. Chem. Theory Comput. 2020, $16,5150-5162$.

[50] Rosa, M.; Micciarelli, M.; Laio, A.; Baroni, S. Sampling Molecular Conformers in Solution with Quantum Mechanical Accuracy at a Nearly Molecular-Mechanics Cost $J$. Chem. Theory Comput. 2016, 12, 4385-4389.

[51] Del Galdo, S.; Chandramouli, B.; Mancini, G.; Barone, V. Assessment of Multi-Scale Approaches for Computing UV-Vis Spectra in Condensed Phases: Toward an Effective 
yet Reliable Integration of Variational and Perturbative QM/MM Approaches J. Chem. Theory Comput. 2019, 15, 3170-3184.

[52] Del Galdo, S.; Fusè, M.; Barone, V. The ONIOM/PMM Model for Effective Yet Accurate Simulation of Optical and Chiroptical Spectra in Solution: Camphorquinone in Methanol as a Case Study J. Chem. Theory Comput. 2020, 16, 3294-3306;

[53] Bondanza, M.; Nottoli, M.; Cupellini, L.; Lipparini, F.; Mennucci, B. Polarizable embedding QM/MM: the future gold standard for complex (bio)systems? Phys. Chem. Chem. Phys. 2020, 14433-14448.

[54] Petrone, A.; Cerezo, J.; Ferrer, F. J. A.; Donati, G.; Improta, R.; Rega, N.; Santoro, F. Absorption and Emission Spectral Shapes of a Prototype Dye in Water by Combining Classical/Dynamical and Quantum/Static Approaches J. Phys. Chem. A 2015, 119, 54265438.

[55] Cerezo, J.; Santoro, F.; Prampolini, G. Comparing classical approaches with empirical or quantum-mechanically derived force fields for the simulation electronic lineshapes: application to coumarin dyes Theor. Chem. Acc. 2016, 135, 143.

[56] Cerezo, J.; Aranda, D.; Avila Ferrer, F. J.; Prampolini, G.; Santoro, F. AdiabaticMolecular Dynamics Generalized Vertical Hessian Approach: A Mixed Quantum Classical Method to Compute Electronic Spectra of Flexible Molecules in the Condensed Phase J. Chem. Theory Comput. 2020, 16, 1215-1231.

[57] Crespo-Otero, R.; Barbatti, M. Spectrum simulation and decomposition with nuclear ensemble: formal derivation and application to benzene, furan and 2-phenylfuran Theor. Chem. Acc. 2012, 131, 1237.

[58] De Vetta, M.; Baig, O.; Steen, D.; Nogueira, J.; González, L. Assessing Configurational Sampling in the Quantum Mechanics/Molecular Mechanics Calculation of Temoporfin Absorption Spectrum and Triplet Density of States Molecules 2018, 23, 2932. 
[59] Hazra, A.; Nooijen, M. Derivation and Efficient Implementation of a Recursion Formula to Calculate Harmonic Franck-Condon Factors for Polyatomic Molecules Int. J. Quantum Chem. 2003, 95, 643-657.

[60] Dierksen, M.; Grimme, S. An efficient approach for the calculation of Franck-Condon integrals of large molecules J. Chem. Phys. 2005, 122, 244101.

[61] Jankowiak, H.-C.; Stuber, J. L.; Berger, R. Vibronic transitions in large molecular systems: Rigorous prescreening conditions for Franck-Condon factors J. Chem. Phys. 2007, $127,234101$.

[62] Santoro, F.; Improta, R.; Lami, A.; Bloino, J.; Barone, V. Effective method to compute Franck-Condon integrals for optical spectra of large molecules in solution J. Chem. Phys. 2007, 126, 084509.

[63] Santoro, F.; Lami, A.; Improta, R.; Bloino, J.; Barone, V. Effective method for the computation of optical spectra of large molecules at finite temperature including the Duschinsky and Herzberg-Teller effect: The Qx band of porphyrin as a case study J. Chem. Phys. 2008, $128,224311$.

[64] Ianconescu, R.; Pollak, E. Photoinduced Cooling of Polyatomic Molecules in an Electronically Excited State in the Presence of Dushinskii Rotations J. Phys. Chem. A 2004, $108,7778-7784$.

[65] Tatchen, J.; Pollak, E. Ab initio spectroscopy and photoinduced cooling of the transstilbene molecule J. Chem. Phys. 2008, 128, 164303.

[66] Huh, J.; Berger, R. Coherent state-based generating function approach for FranckCondon transitions and beyond J. Phys.: Conf. Ser. 2012, 380, 012019.

[67] Borrelli, R.; Capobianco, A.; Peluso, A. Generating Function Approach to the Calculation of Spectral Band Shapes of Free-Base Chlorin Including Duschinsky and HerzbergTeller Effects J. Phys. Chem. A 2012, 116, 9934-9940. 
[68] Baiardi, A.; Bloino, J.; Barone, V. General Time Dependent Approach to Vibronic Spectroscopy Including Franck-Condon, Herzberg-Teller, and Duschinsky Effects J. Chem. Theory Comput. 2013, 9, 4097-4115.

[69] Avila Ferrer, F. J.; Cerezo, J.; Soto, J.; Improta, R.; Santoro, F. First-principle computation of absorption and fluorescence spectra in solution accounting for vibronic structure, temperature effects and solvent inhomogenous broadening Comput. Theoret. Chem. 2014, 1040-1041, 328-337.

[70] Cerezo, J.; Mazzeo, G.; Longhi, G.; Abbate, S.; Santoro, F. Quantum-Classical Calculation of Vibronic Spectra along a Reaction Path: The Case of the ECD of Easily Interconvertible Conformers with Opposite Chiral Responses J. Phys. Chem. Lett. 2016, 7, 4891-4897.

[71] Zuehlsdorff, T. J.; Isborn, C. M. Combining the ensemble and Franck-Condon approaches for calculating spectral shapes of molecules in solution J. Chem. Phys. 2018, $148,024110$.

[72] Zuehlsdorff, T. J.; Napoli, J. A.; Milanese, J. M.; Markland, T. E.; Isborn, C. M. Unraveling electronic absorption spectra using nuclear quantum effects: Photoactive yellow protein and green fluorescent protein chromophores in water J. Chem. Phys. 2018, 149, 024107.

[73] Zuehlsdorff, T. J.; Montoya-Castillo, A.; Napoli, J. A.; Markland, T. E.; Isborn, C. M. Optical spectra in the condensed phase: Capturing anharmonic and vibronic features using dynamic and static approaches J. Chem. Phys. 2019, 151, 074111.

[74] Loco, D.; Cupellini, L. Modeling the absorption lineshape of embedded systems from molecular dynamics: A tutorial review Int. J. Quantum Chem. 2019, 119, e25726.

[75] Cerezo, J.; Ferrer, F. J. A.; Prampolini, G.; Santoro, F. Modeling Solvent Broadening on the Vibronic Spectra of a Series of Coumarin Dyes. From Implicit to Explicit Solvent Models J. Chem. Theory Comput. 2015, 11, 5810-5825. 
[76] Avila Ferrer, F. J.; Santoro, F. Comparison of vertical and adiabatic harmonic approaches for the calculation of the vibrational structure of electronic spectra Phys. Chem. Chem. Phys. 2012, 14, 13549-13563.

[77] Cerezo, J.; Santoro, F. Revisiting Vertical Models To Simulate the Line Shape of Electronic Spectra Adopting Cartesian and Internal Coordinates J. Chem. Theory Comput. 2016, 12, 4970-4985.

[78] Tomasi, J.; Mennucci, B.; Cammi, R. Quantum Mechanical Continuum Solvation Models Chem. Rev. 2005, 105, 2999-3094.

[79] Cacelli, I.; Prampolini, G. Parametrization and Validation of Intramolecular Force Fields Derived from DFT Calculations J. Chem. Theory Comput. 2007, 3, 1803-1817.

[80] Barone, V.; Cacelli, I.; De Mitri, N.; Licari, D.; Monti, S.; Prampolini, G. Joyce and Ulysses: Integrated and User-Friendly Tools for the Parameterization of Intramolecular Force Fields from Quantum Mechanical Data. Phys. Chem. Chem. Phys. 2013, 15, 373651.

[81] Cerezo, J.; Prampolini, G.; Cacelli, I. Developing accurate intramolecular force fields for conjugated systems through explicit coupling terms Theor. Chem. Acc. 2018, 137, 80.

[82] Lax, M. The Franck-Condon Principle and Its Application to Crystals J. Chem. Phys. 1952, 20, 1752-1760.

[83] Baiardi, A.; Bloino, J.; Barone, V. General formulation of vibronic spectroscopy in internal coordinates J. Chem. Phys. 2016, 144, 084114.

[84] Madsen, D.; Christiansen, O.; Norman, P.; König, C. Vibrationally resolved emission spectra of luminescent conjugated oligothiophenes from anharmonic calculations Phys. Chem. Chem. Phys. 2019, 21, 17410-17422.

[85] Law, Y. K.; Hassanali, A. A. The importance of nuclear quantum effects in spectral line broadening of optical spectra and electrostatic properties in aromatic chromophores $J$. Chem. Phys. 2018, 148, 102331. 
[86] Salomon-Ferrer, R.; Case, D. A.; Walker, R. C. An overview of the Amber biomolecular simulation package WIREs Comput. Mol. Sci. 2013, 3, 198-210.

[87] Wang, J.; Wang, W.; Kollman, P. A.; Case, D. A. Automatic atom type and bond type perception in molecular mechanical calculations J. Mol. Graph. Model. 2006, 25, 247 260.

[88] Sousa da Silva, A. W.; Vranken, W. F. ACPYPE - AnteChamber PYthon Parser interfacE BMC research notes 2012, 5, 367.

[89] Nikitin, A. M.; Lyubartsev, A. P. New six-site acetonitrile model for simulations of liquid acetonitrile and its aqueous mixtures J. Comput. Chem. 2007, 28, 2020-2026.

[90] Cacelli, I.; Cerezo, J.; ; De Mitri, N.; Prampolini, G.; JoyCE2.10, a Fortran 77 code for intra-molecular force field parameterization., available free of charge at http://www.pi.iccom.cnr.it/joyce, last consulted July; 2020.

[91] Berendsen, H. J. C.; Postma, J. P. M.; van Gunsteren, W. F.; DiNola, A.; Haak, J. R. Molecular dynamics with coupling to an external bath J. Chem. Phys. 1984, 81, 36843690.

[92] Parrinello, M.; Rahman, A. Polymorphic transitions in single crystals: A new molecular dynamics method J. Appl. Phys. 1981, 52, 7182-7190.

[93] Bussi, G.; Donadio, D.; Parrinello, M. Canonical sampling through velocity rescaling J. Chem. Phys. 2007, 126, 014101.

[94] Frisch, M. J.; Trucks, G. W.; Schlegel, H. B.; Scuseria, G. E.; Robb, M. A.; Cheeseman, J. R.; Scalmani, G.; Barone, V.; Petersson, G. A.; Nakatsuji, H.; Li, X.; Caricato, M.; Marenich, A. V.; Bloino, J.; Janesko, B. G.; Gomperts, R.; Mennucci, B.; Hratchian, H. P.; Ortiz, J. V.; Izmaylov, A. F.; Sonnenberg, J. L.; Williams-Young, D.; Ding, F.; Lipparini, F.; Egidi, F.; Goings, J.; Peng, B.; Petrone, A.; Henderson, T.; Ranasinghe, D.; Zakrzewski, V. G.; Gao, J.; Rega, N.; Zheng, G.; Liang, W.; Hada, M.; Ehara, M.; Toyota, K.; Fukuda, R.; Hasegawa, J.; Ishida, M.; Nakajima, T.; Honda, Y.; Kitao, O.; 
Nakai, H.; Vreven, T.; Throssell, K.; Montgomery, J. A., Jr.; Peralta, J. E.; Ogliaro, F.; Bearpark, M. J.; Heyd, J. J.; Brothers, E. N.; Kudin, K. N.; Staroverov, V. N.; Keith, T. A.; Kobayashi, R.; Normand, J.; Raghavachari, K.; Rendell, A. P.; Burant, J. C.; Iyengar, S. S.; Tomasi, J.; Cossi, M.; Millam, J. M.; Klene, M.; Adamo, C.; Cammi, R.; Ochterski, J. W.; Martin, R. L.; Morokuma, K.; Farkas, O.; Foresman, J. B.; Fox, D. J.; Gaussian 16 Revision C.01; 2016; Gaussian Inc. Wallingford CT.

[95] Ufimtsev, I. S.; Martínez, T. J. Quantum Chemistry on Graphical Processing Units. 1. Strategies for Two-Electron Integral Evaluation J. Chem. Theory Comput. 2008, 4, 222231.

[96] Ufimtsev, I. S.; Martinez, T. J. Quantum Chemistry on Graphical Processing Units. 2. Direct Self-Consistent-Field Implementation J. Chem. Theory Comput. 2009, 5, 10041015 .

[97] Ufimtsev, I. S.; Martinez, T. J. Quantum Chemistry on Graphical Processing Units. 3. Analytical Energy Gradients, Geometry Optimization, and First Principles Molecular Dynamics J. Chem. Theory Comput. 2009, 5, 2619-2628.

[98] Yanai, T.; Tew, D. P.; Handy, N. C. A new hybrid exchange correlation functional using the Coulomb-attenuating method (CAM-B3LYP) Chem. Phys. Lett. 2004, 393, 51 - 57.

[99] Grimme, S.; Ehrlic, S.; Goerigk, L. Effect of the Damping Function in Dispersion Corrected Density Functional Theory J. Comp. Chem. 2011, 32, 1456-65.

[100] Santoro, F.; $\mathscr{F} \mathscr{C}$ classes, a Fortran 77 code for vibronic calculations, visit: http://www.pi.iccom.cnr.it/fcclasses, last consulted November; 2019.

[101] Santoro, F.; Cerezo, J.; $\mathscr{F} \mathscr{C}$ classes3, a code for vibronic calculations. Available upon request; 2019.

[102] Peng, Q.; Niu, Y.; Deng, C.; Shuai, Z. Vibration correlation function formalism of radiative and non-radiative rates for complex molecules Chem. Phys. 2010, 370, 215-222. 
[103] Andreussi, O.; Prandi, I. G.; Campetella, M.; Prampolini, G.; Mennucci, B. Classical Force Fields Tailored for QM Applications: Is It Really a Feasible Strategy? J. Chem. Theory Comput. 2017, 13, 4636-4648.

[104] Chen, Z.; Fimmel, B.; Würthner, F. Solvent and substituent effects on aggregation constants of perylene bisimide $\pi$-stacks - a linear free energy relationship analysis Org. Biomol. Chem. 2012, 10, 5845-5855.

[105] Maity, S.; Shyamal, M.; Mazumdar, P.; Sahoo, G. P.; Maity, R.; Salgado-Morán, G.; Misra, A. Solvatochromism and turn-off fluorescence sensing property of N,N'-bis(3pentyl)perylene-3, 4, 9, 10-bis(dicarboximide) towards nitroaromatics and photophysical study of its microstructures J. Mol. Liq. 2016, 224, 255 - 264.

[106] Prampolini, G.; Ingrosso, F.; Cerezo, J.; Iagatti, A.; Foggi, P.; Pastore, M. Shortand Long-Range Solvation Effects on the Transient UV-Vis Absorption Spectra of a $\mathrm{Ru}(\mathrm{II})-$ Polypyridine Complex Disentangled by Nonequilibrium Molecular Dynamics $J$. Phys. Chem. Lett. 2019, 10, 2885-2891.

[107] Eilmes, A. Solvatochromic probe in molecular solvents: implicit versus explicit solvent model Theor. Chem. Acc. 2014, 133, 1-13.

[108] Cerezo, J.; Petrone, A.; Ferrer, F. J. A.; Donati, G.; Santoro, F.; Improta, R.; Rega, N. Electronic spectroscopy of a solvatochromic dye in water: comparison of static cluster/implicit and dynamical/explicit solvent models on structures and energies Theor. Chem. Acc. 2016, 135, 263-275.

[109] Avila Ferrer, F. J.; Cerezo, J.; Stendardo, E.; Improta, R.; Santoro, F. Insights for an Accurate Comparison of Computational Data to Experimental Absorption and Emission Spectra: Beyond the Vertical Transition Approximation J. Chem. Theory Comput. 2013, 9, 2072-2082. 
[110] Diez-Cabanes, V.; Prampolini, G.; Francés-Monerris, A.; Monari, A.; Pastore, M. Iron's Wake: The Performance of Quantum Mechanical-Derived Versus General-Purpose Force Fields Tested on a Luminescent Iron Complex Molecules 2020, 25, 3084;

[111] Milanese, J. M.; Provorse, M. R.; Alameda, E.; Isborn, C. M. Convergence of Computed Aqueous Absorption Spectra with Explicit Quantum Mechanical Solvent J. Chem. Theory Comput. 2017, 13, 2159-2171;

[112] Chiariello, M. G.; Donati, G.; Rega, N. Time-Resolved Vibrational Analysis of Excited State Ab Initio Molecular Dynamics to Understand Photorelaxation: The Case of the Pyranine Photoacid in Aqueous Solution J. Chem. Theory Comput., https://doi.org/10.1021/acs.jctc.0c00810;

[113] Marenich, A. V.; Cramer, C. J.; Truhlar, D. G. Electronic Absorption Spectra and Solvatochromic Shifts by the Vertical Excitation Model: Solvated Clusters and Molecular Dynamics Sampling J. Phys. Chem. B 2015, 119, 958-967;

[114] Raucci, U.; Perrella, F.; Donati, G.; Zoppi, M.; Petrone, A.; Rega, N. Ab-initio molecular dynamics and hybrid explicit-implicit solvation model for aqueous and nonaqueous solvents: GFP chromophore in water and methanol solution as case study J. Comput. Chem. 2020, 41, 2228-2239.

[115] Loco, D.; Gelfand, N.; Jurinovich, S.; Protti, S.; Mezzetti, A.; Mennucci, B. Polarizable QM/Classical Approaches for the Modeling of Solvation Effects on UV-Vis and Fluorescence Spectra: An Integrated Strategy J. Phys. Chem. A 2018, 122, 390-397.

[116] Giovannini, T.; Riso, R. R.; Ambrosetti, M.; Puglisi, A.; Cappelli, C. Electronic transitions for a fully polarizable QM/MM approach based on fluctuating charges and fluctuating dipoles: Linear and corrected linear response regimes J. Chem. Phys. 2019, 151, 174104.

[117] Köppel, H.; Domcke, W.; Cederbaum, L. In Conical Intersections, Electronic Structure, Dynamics and Spectroscopy; World Scientific Publishing Co. Singapore; pp 323-368. 
[118] Liu, Y.; Martínez Fernández, L.; Cerezo, J.; Prampolini, G.; Improta, R.; Santoro, F. Multistate coupled quantum dynamics of photoexcited cytosine in gas-phase: Nonadiabatic absorption spectrum and ultrafast internal conversions. Chem. Phys. 2018, 515, 452-463. 